\title{
The role of strength defects in shaping impact crater planforms
}

\author{
W. A. Watters, L. M. Geiger, M. Fendrock, R. Gibson, C. Hundal \\ wwatters@wellesley.edu; Whitin Observatory, Department of Astronomy, Wellesley College, 106 Central St., Wellesley, \\ MA, USA, 02481
}

\begin{abstract}
High-resolution imagery and digital elevation models (DEMs) were used to measure the planimetric shapes of well-preserved impact craters. These measurements were used to characterize the size-dependent scaling of the departure from circular symmetry, which provides useful insights into the processes of crater growth and modification. For example, we characterized the dependence of the standard deviation of radius $\left(\sigma_{R}\right)$ on crater diameter $(D)$ as $\sigma_{R} \sim D^{m}$. For complex craters on the Moon and Mars, $m$ ranges from 0.9 to 1.2 among strong and weak target materials. For the martian simple craters in our data set, $m$ varies from 0.5 to 0.8 . The value of $m$ tends toward larger values in weak materials and modified craters, and toward smaller values in relatively unmodified craters as well as craters in high-strength targets, such as young lava plains. We hypothesize that $m \approx 1$ for planforms shaped by modification processes (slumping and collapse), whereas $m$ tends toward $\sim 1 / 2$ for planforms shaped by an excavation flow that was influenced by strength anisotropies.

Additional morphometric parameters were computed to characterize the following planform properties: the planform aspect ratio or ellipticity, the deviation from a fitted ellipse, and the deviation from a convex shape. We also measured the distribution of crater shapes using Fourier decomposition of the planform, finding a similar distribution for simple and complex craters. By comparing the strength of small and large circular harmonics, we confirmed that lunar and martian complex craters are more polygonal at small sizes. Finally, we have used physical and geometrical principles to motivate scaling arguments and simple Monte Carlo models for generating synthetic planforms, which depend on a characteristic length scale of target strength defects. One of these models can be used to generate populations of synthetic planforms which are very similar to the measured population of well-preserved simple craters on Mars.
\end{abstract}

Keywords:

\section{Introduction}

The present study is concerned with the planimetric shape of impact craters, otherwise known as the "planform". The "cavity planform" is the shape of an elevation contour near the base of the rim, whereas "rim planform" refers to the vertically-projected trace of the crater rim crest. Previous work has identified three factors which influence the shape of crater planforms: (1) the angle of impact with respect to the horizontal; (2) strength heterogeneities in the target, such as fractures and faults; and (3) preexisting topography (Gifford and Maxwell, 1979; Eppler et al., 1983; Gulick et al., 2008). The primary focus of the present study is to understand the influence of (2), while presenting measurements that are to some extent influenced by (1). We do not Preprint submitted to Icarus

December 1, 2016

(C) 2016. This manuscript version is made available under the Elsevier user license

http://www.elsevier.com/open-access/userlicense/1.0/ 
directly address (3), but have largely excluded from our study craters that formed on preexisting features with significant topographic expression.

\subsection{Elongation from oblique impact}

The first description of the planimetric shape of impact craters was Galileo's remark about a "perfectly round" cavity in his first telescopic views of the Moon, recorded in the Sidereus Nuncius of 1610. Formal experiments conducted in the late 20th century century showed that elongated craters form in high-speed impacts with impact angles of less than $5^{\circ}$ from the horizontal (Gault and Wedekind, 1978). The relationship between planform elongation, impact angle, and the excavation flow have been examined more recently in detailed experiments; e.g., Anderson et al. (2004b) found that the excavation flow is asymmetric for impact angles as high as $30^{\circ}$ from the horizontal, although the final crater planform is highly symmetric nonetheless. Numerical modeling studies have examined the transition from symmetric to elliptical shapes, finding that this can be explained in terms of a transition to ricochet and grazing impacts, in which momentum-driven displacement becomes more significant and the projectile is more affected by brittle fracture than shock compression (Elbeshausen et al., 2013).

Morphometric studies have reported the ellipticity of large numbers of crater planforms, defined as the ratio of the maximum to minimum crater diameter (Bottke et al., 2000). This work found that less than $5 \%$ of the population of well-preserved craters exhibit an ellipticity exceeding 1.1, which is approximately consistent with the expected distribution of impact angles below $12^{\circ}$ and consistent with results of numerical simulations (Collins et al., 2011). A more recent survey of northern-hemisphere impact craters on Mars estimated that 3\% (Barlow, 2015) of craters show signs of oblique impact. This leaves the vast majority of crater planforms whose relatively slight departure from symmetry is accounted for by other effects. Among the most important of these is the influence of strength defects in target materials.

\subsection{Asymmetry from strength defects}

At the dawn of the space age, the most significant insights regarding the effect of strength heterogeneities on planform shape were derived from the study of terrestrial craters. Gene Shoemaker was the first to suggest that strength anisotropies may have created the squarish rim planform of Barringer Crater (Shoemaker, 1960). Shoemaker observed that the regional pattern of tectonic lineaments is approximately aligned with the diagonals of the square planform. At least two subsequent studies have supported the conclusion that pre-impact joints are aligned with the diagonals of the square rather than its sides. First, Roddy (1978) measured pre-impact joints in the thin Moenkopi stratum and the much thicker Kaibab carbonate stratum from which the crater is mostly excavated, finding that these align within $6^{\circ}$ of the diagonals of the planform. Later, Kring (2015) confirmed the alignment of diagonals with joints in the Moenkopi. Poelchau et al. (2009) proposed that the square planform results from a minimum of shear strength in the direction of pre-impact fracture alignments (see also Watters et al. (2011)).

Nonetheless, a detailed recent study of pre-impact fractures at Barringer Crater came to the opposite conclusion, finding that pre-impact fractures align more nearly with the crater walls (Kumar and Kring, 2008). This study suggested that the squarish crater planform is the consequence of the excavation flow tending to overturn materials along axes that are parallel to planes of weakness. A third model suggests that crater walls should be parallel to pre-impact fractures because of thrust faulting that exploits these preexisting defects (Öhman et al., 2008). Support for the idea that pre-impact fractures are parallel to straight walls in simple craters derives 
from observed alignments with regional joint and fault patterns. Such alignments were found by Öhman et al. (2008) and Beddingfield et al. (2016), although neither study separately reported the distribution of straight wall orientations for morphologically fresh simple craters. The present study and previous work (Watters, 2009) show examples of heavily-modified simple craters with straight walls that align with extensive fractures in the surrounding surface (see also Figure 12 in Öhman et al. (2008)). While at least one laboratory impact experiment found that preexisting fractures bisected crater corners (Gault et al., 1968), large-scale explosion experiments found support for both relationships in different experiments (Fulmer and Roberts, 1963).

A structural study of Endurance crater at Meridiani Planum on Mars $(D=150 \mathrm{~m})$ recently made several observations that are consistent with Shoemaker's original conclusion (Watters et al., 2011). First, the structure of Endurance crater exhibits preservation of the hinge in corners of the planform and destruction of the hinge in between corners, as found at Barringer Crater. In both cases, this implies a concave planform for the transient crater, where excavation proceeded farther in the corners of the planform. Second, the widened fractures in the surrounding target rocks at Endurance crater are dominantly aligned with the diagonals of the square component of the rim planform. Third, the published planform and regional joint azimuths for Tswaing Crater near Pretoria in South Africa exhibit a similar relationship (Brandt and Reimold, 1995; Watters et al., 2011).

In summary, there appears to be a combination of processes through which strength defects may influence the planforms of simple craters and more work is needed before this is entirely understood. By contrast, complex crater planforms are broadly agreed to result from the collapse of transient cavity walls as a consequence of acoustic fluidization (Melosh, 1979) and localized strain and dynamic fault weakening (Senft and Stewart, 2009). Unlike simple craters, complex craters tend to exhibit terraced walls and relatively flat floors, often with central peaks and pits, or peak rings (Melosh, 1989). It was suggested decades ago that preexisting planes of weakness influence the cavity collapse (e.g., Schultz (1976), Eppler et al. (1983)).

A series of papers have examined the degree of alignment between the straight wall segments of complex craters and regional lineaments, concluding that dominant directions of complex crater walls are aligned with large-scale preexisting planes of weakness formed by tectonic stresses. These relationships have been investigated on Venus (Aittola et al., 2007, 2010), Mars (Öhman et al., 2005; Öhman et al., 2006, 2008), and Saturn's Moon Dione (Beddingfield et al., 2016). Some of these studies separately accounted for the preservation state of craters. For example, Öhman et al. (2006) grouped craters into three categories according to preservation, finding that there was no significant difference between the dominant alignments of straight wall segments in these categories. This finding is similar to that of Eppler et al. (1983), who found that the strength of low-order circular harmonics in the rim planform was similar between preservation states in lunar complex craters (i.e., the "polygonality" of complex craters is largely unaffected by long-term modification). A recent survey of polygonal impact craters on Mercury found large regions in which polygonal complex craters are curiously absent (Weihs et al., 2015).

\subsection{Methods for characterizing departures from symmetry}

A large number of quantities have been proposed for characterizing the shape of crater planforms and the departure from circular symmetry. These fall dominantly into three categories. First are (i) measurements of radial deviation: estimates of the departure from circular symmetry through measurement of average deviations from the uniform radius of a circle (Murray and Guest, 1970; Oberbeck et al., 1972). The great advantage of such measurements is that they are straightforward to apply and provide a robust estimate of the overall departure from radial 
symmetry. A second category is concerned with (ii) reducing the planform to a sum of basis functions, such as in a Fourier decomposition of the planform into circular harmonics (Eppler et al., 1977; Eppler et al., 1983). This approach provides significant information about the planform shape rather than just the departure from radial symmetry.

A third category (iii) is concerned with comparing the perimeters or areas of: e.g., the measured planform, circles inscribed within and circumscribed by the measured planform, or circles with perimeters or areas equivalent to those of the measured planform (e.g., Ronca and Salisbury (1966), Murray and Guest (1970), Pike (1977)). These parameters are often termed the "circularity," "circularity ratio," or "circularity index," where a perfectly circular shape has a value of 1 and noncircular shapes have a value $<1$. The current study is concerned with measurements belonging to categories (i) and (ii) since (iii) is most interesting in the case of highly irregular shapes with complex concave outlines, such as ejecta blankets (Calef et al., 2009). More importantly, however, measures of circularity are typically less robust when compared with measures of average radial deviation. For example, supposing the planform encloses an area $A$, if circularity is defined as the perimeter of a circle with area $A$ divided by the measured planform perimeter (as in Baker and Head (2013)), the result will be sensitive to the resolution of the measurement (Mandelbrot, 1983). If circularity is defined as the ratio of the area of the inscribed circle to that of the circle circumscribing the planform (as in Pike (1977)), a single "notch" or excursion in an otherwise circular planform will completely determine the result; i.e., circularity is a poor measure of the overall or average departure from symmetry in some cases.

\subsection{Influence of size, terrain, and modification}

Early high-resolution images of the lunar surface revealed that large craters exhibit a wide variation in planform shape. Some early studies measured the dependence of this variation on diameter (Ronca and Salisbury, 1966; Murray and Guest, 1970; Pike, 1977). A primary feature of this size dependence is illustrated in Figure $1 \mathrm{~A}-\mathrm{C}$, showing three well-preserved martian impact craters in HiRISE images (High-Resolution Imaging Science Experiment on the Mars Reconnaissance Orbiter; McEwen et al. (2010)). Part A shows the HiRISE image of a fresh, small primary crater $(D=50 \mathrm{~m})$ which formed within several years of image acquisition, and which exhibits a somewhat irregular rim planform. The much larger bowl-shaped crater in Figure 1B with diameter $D=1.4 \mathrm{~km}$ has a relatively symmetric, circular outline. Complex craters, by contrast, tend to exhibit highly irregular shapes at even larger diameters (Figure 1C). This tendency for impact craters to become more circular with increasing size, and then depart from circular shape for diameters exceeding the simple-complex transition diameter, was first quantitatively characterized in detail by Pike (1977) for impact craters on the Moon.

The most comprehensive study of the morphometry of lunar complex crater planforms were those of Eppler et al. (1977) and Eppler et al. (1983), in which a Fourier decomposition of the rim planform was used to characterize shape as a function of size, terrain type, and modification state. This work found that low-order harmonics dominate at small sizes and that high-order harmonics are the most influenced by modification processes. In a similar vein, recent morphometric studies involving measurements from orbiter imagery have focused on the distribution and size-dependence of so-called polygonal impact craters (PICs) on all the terrestrial planets (Öhman et al., 2005; Öhman et al., 2006; Aittola et al., 2007; Öhman et al., 2008; Aittola et al., 2010; Öhman et al., 2010). These craters exhibit long, straight wall segments, and are most abundant at diameters slightly exceeding the simple-to-complex transition diameter. An exhaustive summary of the literature concerning PICs can be found in Öhman et al. (2010). 

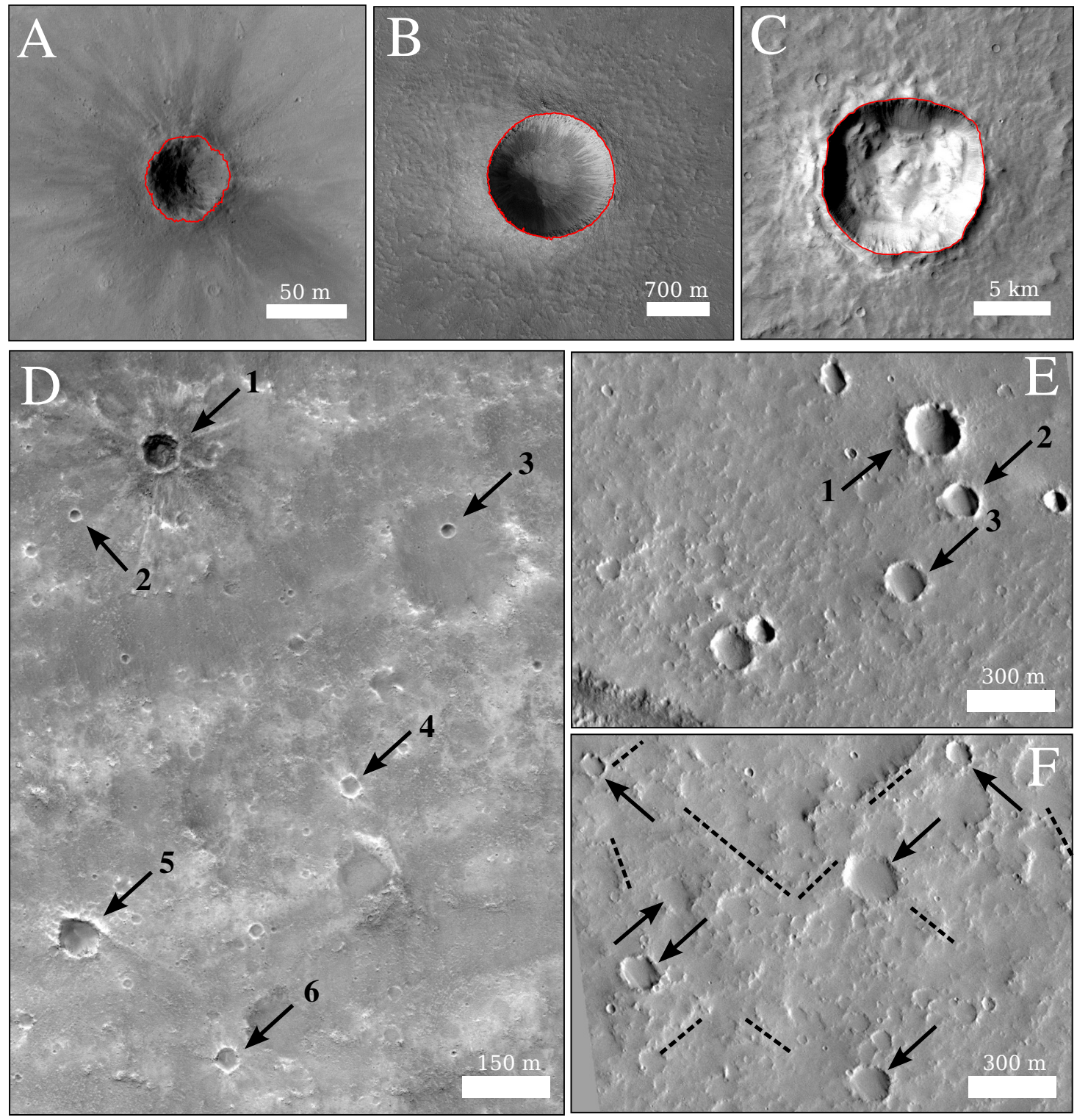

Figure 1: Impact craters on Mars illustrating significant variation in rim planform as a function of size, terrain type, and preservation state. (A) Small simple crater $(D=50 \mathrm{~m})$ with irregular planform that formed within three years of image acquisition (HiRISE ESP_011425_1775). (B) Larger well-preserved simple crater $(D=1.4 \mathrm{~km})$ with highly symmetric planform (HiRISE ESP_012857_1910). (C) Well-preserved complex crater that exhibits a squarish planform (C10-000426 in Robbins and Hynek (2012), HRSC image H1037_0000_Nd4). The rim traces for A-C are shown in red. (D) Simple impact craters exhibiting a wide range of planform shapes according to context terrain and preservation state; crater 1 exhibits the best preservation and a polygonal planform, while 2 and 3 lack obvious ejecta, and formed in smoother terrain, and have relatively symmetric planforms; highly modified craters 4-6 formed in the textured terrain and exhibit relatively polygonal planforms (floor of channel into the Aram Chaos; HiRISE ESP_037782_1825). Parts (E) and (F) show simple craters whose planforms tend toward a rectangular shape as a result of modification controlled by the local joint pattern, as indicated by scarps highlighted by adjacent dashed lines (Watters (2009); cracked plains in Lunae Planum west of Echus Chasma; Mars Orbiter Camera image MGS-MOC/S0400280 (Malin and Edgett, 2001)). 
Since the advent of high-resolution imagery from spacecraft cameras at Mars and the Moon in the past two decades, comparatively little attention has focused on the planform shape of simple impact craters. Calef et al. (2009) used HiRISE imagery to measure and compare the planforms of small-sized well-preserved primary craters $(D<50 \mathrm{~m})$ to those of well-preserved distal secondary craters $(D<150 \mathrm{~m})$. This work did not find a significant difference between the statistical distribution of the circularities of rim planforms, while noting that the most asymmetric craters were all secondaries. This work also measured a slight increase in radial symmetry of rim planforms with increasing diameter for $D<150 \mathrm{~m}$, and a corresponding increase in ejecta planform symmetry.

Figure 1D-F illustrates some primary features of the dependence of planform shape on geologic setting and modification. Part D of this figure shows an unusually complex and intimate mixture of terrains (smooth vs. textured) on the floor of a channel which intersects the Aram Chaos. The crater with the best ejecta preservation forming in the textured terrain (D1) exhibits a highly irregular planform. By contrast, craters that formed in the smooth terrains exhibit a relatively symmetrical planform (D2 and D3). If these have formed in sand-filled depressions, the increase in symmetry is easily understood in terms of an absence of strength defects exploited by excavation and modification processes (Fulmer and Roberts, 1963).

Highly modified craters in the textured terrains in Figure 1D also exhibit highly asymmetric planforms (D4-D6). A region west of Echus Chasma and southwest Lunae Planum contains a prominent conjugate set of joints where it is possible to illustrate how modification can, in some cases, enhance the asymmetry of rim planforms at small diameters (Watters, 2009). Craters with the best preservation in this setting (inferred from the deeper cavities implied by longer shadow lengths) appear to exhibit relatively rounded rim planforms (E1 and E2), while shallower, modified craters assume a rectangular shape with a consistent orientation (E3 and other craters in $\mathrm{E}$ and F). The joints in this landscape are indicated by the shallow scarps highlighted with dashed lines. Bearing in mind these modification effects, we focus our efforts in this study on globally-derived populations of craters that exhibit signs of good preservation. The influence of geological target strength is also addressed.

This paper is organized as follows. In Section 2 we describe the database of well-preserved martian and lunar impact craters examined in this study. The following section (Section 3) is concerned with the methods used to extract the planforms from images and elevation models, as well as the morphometric parameters computed to characterize planform asymmetry and overall shape. In Section 4 we report on the size-dependent scaling of deviations from symmetry as well as the overall distributions of the values of several other morphometric parameters and the dependence on terrain type. In Section 5 we develop scaling arguments and Monte Carlo models of synthetic crater planforms, which are compared with our measurements in Section 6. This is followed by a discussion in Section 7.

\section{Crater databases and crater attributes}

Our measurements derive from three populations of well-preserved impact craters. Through the rest of this paper and in the supplementary tables, we use the label "LROC-complex" to refer to the set of well-preserved lunar complex craters examined in this study. The rim planforms of craters in LROC-complex were measured manually from a global LROC WAC mosaic (Lunar Reconnaissance Orbiter Wide-Angle Camera, Robinson et al. (2010); Robinson (2011)), as described in the following section. These craters are globally distributed on the lunar surface, and were identified in the catalog published in Losiak et al. (2009) and determined by Kalynn 
et al. (2013) to show signs of good preservation, including sharp rims, well-defined ejecta, and a paucity of overlapping craters. We have restored to this population the craters discarded by Kalynn et al. (2013) because they exhibit "irregular shapes," since the variation in shape is part of what the present study aims to characterize. LROC-complex contains 112 craters ranging in diameter from 19 to $150 \mathrm{~km}$.

The label "HRSC-complex" refers to a globally-distributed population of 382 complex craters on Mars that are completely visible in orthorectified, nadir-pointing images acquired by the HighResolution Science Camera (HRSC) on Mars Express published by the time our study began (Jaumann et al., 2007). All the craters in HRSC-complex were identified in Robbins and Hynek (2012) as belonging to their class 4 preservation state, meaning they possess a sharp rim or "pristine" interior or ejecta morphology or relatively deep cavity. The craters in this population range in diameter from roughly 5 to $40 \mathrm{~km}$. The rim planforms were traced manually, as described in the following section.

Finally, the label "HiRISE-simple" is used to refer to a database of 364 simple craters on Mars. This database is a somewhat expanded version of the database addressed in a former paper that reported on other morphometric quantities (Watters et al., 2015). All craters in HiRISE-simple are visible in HiRISE stereo imagery, which at the time of writing has yet to cover more than $1 \%$ of the planet surface. These craters range in diameter from $25 \mathrm{~m}$ to $5.5 \mathrm{~km}$. Craters in HiRISEsimple were selected because they exhibit signs of relatively good preservation with respect to other craters in the same image (if any), or were selected because they exhibit signs of good preservation as the only crater in the scene. As such, these craters nevertheless exhibit a range of preservation states, where this has been assessed using the schema described in Watters et al. (2015); we refer the reader to that prior work for details and summarize the approach in the next paragraph. Note that prior schemes for assessing the preservation state of complex craters, which rely partly on rim sharpness, could not be used (Barlow, 2004; Robbins and Hynek, 2012) because even very fresh simple craters with $D<1 \mathrm{~km}$ often exhibit rounded rims (Watters et al., 2015).

In summary, a "modification index" $\left(i_{M}\right)$ ranging from -3 (best preservation) to +6 (most modified) was assigned to each crater based on qualitative features including the size of any sediment body inside the cavity and the condition of ejecta preservation. Craters were subsequently grouped into classes MV1, MV2, and MV3 according to their value of $i_{M}$, where MV1 indicates excellent preservation whereas MV3 suggests significant modification (MV here means "modification state from visual inspection"; see Watters et al. (2015)). Additionally, craters were grouped into these semi-quantitative categories: (i) Moderately modified (MM) if the crater did not belong to MV3 and rim height/rim diameter $(h / D)$ exceeded the average minus one standard deviation, and rim-to-floor cavity depth/rim diameter $(d / D)$ exceeded the average minus one standard deviation; (ii) Least Modified (LM) is a subset of MM for which $h / D$ and $d / D$ exceed their respective average values for the population as a whole, while belonging to MV1; (iii) Highly Modified (HM) were all those not in MM. Craters that occur in large clusters and prominent crater rays have been excluded from the database (Watters et al., 2015).

The lunar craters have been assigned to two terrain types: "strong" (formed in maria) or "weak" (formed in highlands) by inspection of LROC-WAC imagery. Unfortunately, there are just 25 maria craters in our database. For the martian craters, we assigned terrain types according to the approach described in Watters et al. (2015) (see Table 2). That is, we used the Tanaka et al. (2014) geological map to determine whether craters formed in terrains dominated by lava plains and flows, which are relatively strong materials. The label "strong I" applies to a superset of units AHv, eHv, 1Av, 1Avf and lHv in Tanaka et al. (2014). The subset labeled "weak I" is the comple- 
ment of "strong I." The label "weak II" refers to coarse-grained deposits and fractured targets: units comprising crater interiors and ejecta, drop moraines, mass wasting products, and catastrophic debris flow deposits. In particular, "weak II" is a superset of units Aa, AHi, Ht, Hto, 1Aa. Images from the Thermal Emission Imaging System on the Mars Odyssey Spacecraft (THEMIS, Christensen et al. (2004)) and HiRISE images were used to identify crater floors and ejecta on a smaller scale than was mapped by Tanaka et al. (2014) to assign craters to "weak II". Also, craters that contributed to the AHi unit (Amazonian impact unit) were instead assigned to the unit dominating their immediate context. The subset of "strong II" is defined as the complement of "weak II'.

The positions, diameters, attributes, and morphometric parameters of all craters in the three databases (HiRISE-simple, HRSC-complex, and LROC-complex) are supplied in tables of comma-separated values as part of the online supplement.

\section{Methods}

In this section, we describe how crater planforms were measured and then define morphometric quantities computed from these. First, the crater planforms were extracted from orbiter images using two methods: (a) manual tracing of crater rims from spacecraft images, as well as (b) semi-automated extraction of rim-crest pixels from stereo-derived elevation models using machine vision tools developed in-house and described in Geiger (2013) and Watters et al. (2015).

\subsection{Manual tracing of complex crater rims}

The rim planforms of the complex craters examined in this study were manually traced from images. We used a manual trace instead of digital elevation models for two reasons. First, at the time this work was conducted, no example of the latter products provided the required resolution and coverage. For example, LOLA-derived topography contains large null-data gaps between altimeter tracks near the equator, which are larger than the deviations from symmetry that we sought to measure in this work. Second, well-preserved complex craters tend to exhibit very sharp rims that are clearly expressed and readily traceable in most images. Nevertheless, it is important to bear in mind that shadows and other illumination effects can influence the apparent trace of crater rims; we cannot rule out that some of our rim planforms have been distorted in this way (Schultz, 1976; Öhman et al., 2006).

The largest source of uncertainty in tracing complex crater rims derives from the occasional presence of multiple rim candidates, the result of terracing that is a hallmark of complex craters. We adopted the following definition and convention in order to identify rims in an objective and repeatable fashion: the complex crater rim is the topographic ridge, highlighted by an illumination contrast, that encompasses the largest portion of the crater cavity while exhibiting the smallest total radial discontinuity. That is, in tracing crater rims, we optimized two factors: (a) the amount of the cavity that is encompassed by the rim, as well as (b) the extent to which the rim is contiguous, reducing as far as possible the total length of radial breaks.

The consequences of the uncertainty introduced by complex crater terracing is mitigated by the statistical nature of this study. First, as discussed in Section 3.3, we have chosen the standard deviation of radius as the most important measure of the departure from circular symmetry, which will not be sensitive to errors that occur over a small fraction of the entire circumference. Second, we have made these measurements for a large number of craters on two worlds; even significant 
errors affecting individual craters will not significantly influence the average characteristics of the population as a whole.

Rims were manually traced using QGIS, a free geographical information system (GIS) program available for many computing platforms. Pixels were selected along the rim wherever it appears to deviate from a straight-line path: i.e., along a short straight wall segment, only the end-points were sampled. Traces were later interpolated and resampled at regular intervals along straight-line paths, as discussed in the next section, so that positions between manually-selected points would receive equal weight. Because complex crater diameters in LROC-complex and HRSC-complex span roughly an order of magnitude, the rims of the largest craters were traced at a resolution somewhat coarser than the image resolution to avoid collecting far more information than is useful to the analysis. In all cases, the image pixel size is small compared with the length scale of deviations from circular symmetry. For craters in HRSC-complex, the range of image resolutions was $12 \mathrm{~m}$ to $100 \mathrm{~m}$, while the overall deviation from symmetry (maximum radius - minimum radius) ranged from $191 \mathrm{~m}$ to $7 \mathrm{~km}$. For craters in LROC-complex, the image resolution was roughly $100 \mathrm{~m} / \mathrm{pixel}$, while the overall deviation ranged from $1.1 \mathrm{~km}$ to $31.7 \mathrm{~km}$.

Craters in LROC-complex were cropped from the LROC WAC mosaic of Robinson (2011) and reprojected with crater-centered zero latitude to avoid projection-related distortions. Craters in HRSC-complex were measured from the orthorectified HRSC nadir-pointing images which are supplied with HRSC DEM products Jaumann et al. (2007) and were also reprojected. To examine the effect of reprojecting the images, we performed our entire analysis on the HRSC planforms after restoring them to the projection of the original products. As discussed in Section 4.2, this had a barely-noticeable effect on the results.

\subsection{Semi-automatic tracing of simple crater rims}

Whereas well-preserved complex craters commonly present well-defined rims that can be confidently traced in imagery, this is often not the case for small, well-preserved simple craters, whose rims are typically at least somewhat rounded (Watters et al., 2015). For this reason, we have used high-resolution DEMs to measure the properties of simple crater rim planforms and cavity planforms.

All planforms in HiRISE-simple were traced in a mostly automatic fashion using free and open source tools, along with scripts developed in-house according to the methods described in Geiger (2013) and Watters et al. (2015). We refer the reader to this former work for details and summarize the main steps here. First, the HiRISE images were normalized, equalized, projected, and mosaicked using the Integrated Software for Imagers and Spectrometers (ISIS, Anderson et al. (2004a)). Digital elevation models were generated from these images using the Ames Stereo Pipeline 2.0 (ASP, Moratto et al. (2010)). The models are map-projected with the crater at the central latitude of the projection to avoid projection-related distortions. The models were then automatically filtered to remove artifactual steps and islands of spurious elevations that sometimes occur in shadows and patches of featureless terrain. In some cases, the shadows were completely masked before the feature extraction step described below.

Python scripts written in-house were used to identify positions belonging to the crater rim crest by first sampling the elevations along radial elevation profiles. Points-of-interest (POIs) were identified by the program as being (a) local elevation maxima or (b) the global maximum for each profile and (c) major breaks in slope. The program then assembles a composite rim by tracing the global maxima and bridging gaps with local maxima as needed, while minimizing the total length of radial discontinuities. The resulting rim trace is sampled at the same resolution as the DEM. The method is semi-automatic because each trace is then manually inspected and 
any errors are corrected by substituting a manually-traced fragment that is "snapped" to POIs originally identified by the program, including major breaks in slope where necessary. This refinement process was required in roughly $10 \%$ of craters.

The vertically-projected trace of the rim crest is the "rim planform." For craters in HiRISEsimple only, we also measured a "cavity planform." This is defined as an elevation contour measured near the lowest elevation along the 3-D trace of the rim crest. To be precise, this contour was measured at an elevation of $0.95 d_{\min }$, where $d_{\min }$ is the vertical distance from the crater floor to the minimum rim crest elevation. In this way, we ensure that the cavity planform is not influenced by small irregularities that are otherwise introduced by tracing a contour near a breach in the crater rim.

The stereo correlation reliably obtains elevations along the rim crest because this feature presents a highly distinctive boundary that is easily matched between images in a stereo pair. By contrast, shadowed regions of the crater wall are nearly featureless and the ASP will sometimes return no-data values in these areas. As a result, whereas the "rim planform" is nearly always extracted intact using our process, the cavity planform is sometimes impossible to measure without first interpolating over shadows in the elevation model. Because this interpolation can significantly influence the cavity planform's overall shape, we have opted to discard cavity planforms where there is a no-data gap on account of shadowing. As a result, we have measured significantly fewer cavity planforms than rim planforms, discarding $18 \%$ of the former. From the 364 craters in HiRISE-simple, rim planforms were successfully extracted from 352 and cavity planforms from 299.

Finally, the orientation of the elevation model can also influence the shape of the cavity planform. It is therefore important to stress that our elevation models are not in any sense tied to MOLA topography. Instead, a plane was fit to the rim crest elevations and subtracted from the elevation model as a whole. If the rim elevations for a single crater are highly asymmetric, this could introduce an elongation distortion to the cavity planform. We have specifically avoided craters that form in small clusters of comparable age (indicated by similar state of preservation), in order to exclude low-velocity secondaries which may exhibit a significant asymmetry in rim elevation.

\subsection{Morphometric parameters}

Many of the parameters defined in this section are listed in Table 1 and illustrated in Figure 2. We measured morphometric parameters to characterize (i) the planform's overall departure from radial symmetry (from a circle) and (ii) to characterize the shape. In the former case we have used the "radial deviation," defined simply as the standard deviation of crater radius. This is measured by fitting a circle to the crater planform to find the geometric center, and then measuring the radii $R_{i}$ of all points belonging to the planform, and then computing their standard deviation. That is,

$$
\sigma_{R} \equiv \sqrt{\frac{1}{N} \sum_{i=1}^{N}\left(R_{i}-\bar{R}\right)^{2}}
$$

where $\bar{R}$ is the average radius. In the online supplementary materials, we have also reported the maximum radial deviation or radial span, $\Delta R$, defined as the maximum minus minimum radius. Unlike $\sigma_{R}$, this quantity is determined by, and hence highly sensitive to, the extrema. We have measured $\sigma_{R}$ instead of an average absolute deviation of the radius from the mean (as in Murray and Guest (1970)) because statistical scaling relations can be used to make specific predictions 
Table 1: Morphometric parameters computed from impact crater planforms in this study.

\begin{tabular}{lll} 
Symbol & Name & Definition \\
\hline$D$ & Planform diameter & average diameter estimated as $2 \bar{R}$ \\
$\bar{R}$ & Planform radius & average of radii measured from a single planform \\
$\sigma_{R}$ & Radial deviation & standard deviation of radii measured from a single planform; eq. (1) \\
$a / b$ & Ellipticity & ratio of major to minor axis of ellipse fitted to planform \\
$\sigma_{E}$ & Residual deviation & root mean square of radial difference between planform and fitted ellipse \\
$\sigma_{C}$ & Concave deviation & root mean square of radial difference between planform and convex hull \\
$\sigma_{E} / \sigma_{R}$ & Residual fraction & residual deviation as a fraction of overall radial deviation \\
$\sigma_{C} / \sigma_{R}$ & Concave fraction & concave deviation as a fraction of overall radial deviation \\
$A_{n}^{*}$ & $\mathrm{n}^{\text {th }}$ harmonic amplitude & radius-normalized magnitude of harmonic $n: A_{n}^{*} \equiv\left|A_{n}\right| / \bar{R}$ \\
$\Omega$ & small harmonic fraction & fraction of total power in small harmonics; eq. $(3)$ \\
$n_{0}$ & Dominant harmonic & harmonic with largest amplitude (e.g., $n_{0}=3 \leftrightarrow$ triangle) \\
$\rho_{R h}$ & Radial rim height correlation & Pearson correlation coefficient for rim planform radius vs. rim height \\
\hline
\end{tabular}

about the scaling of this quantity with radius (see Section 5). That is, we chose standard deviation as the more useful characterization of the distribution of crater radii because it can be directly compared to model predictions. For completeness, we have also computed the average absolute radial deviation, defined as the average of the absolute difference between each radius and the mean radius; these measurements are reported in the online supplement. We have done likewise for the other root mean square (RMS) quantities computed in this paper $\left(\sigma_{E}\right.$ and $\sigma_{C}$, defined below).

Standard deviation is commonly calculated to estimate the uncertainty or variation of a measurement; it is less clear how to estimate the variation or uncertainty of a standard deviation itself. To characterize the uncertainty of the radial deviation of an individual planform, we have performed a bootstrap analysis (Press et al., 1988). This was accomplished by sampling with replacement from the set of radii $R_{i}$ measured from a single crater planform, up to the number that were measured (dictated by the resolution, as discussed in Section 3.2). These samples are taken 10,000 times and $\sigma_{R}$ was computed in each case. The range of the 10,000 values of $\sigma_{R}$ in each case is indicated by the error bars in Figure 3A-C. Ongoing work will examine how correlations between these radii influence this estimate of the uncertainty of $\sigma_{R}$. This estimate of the uncertainty is intended to represent the variation of $\sigma_{R}$ in a hypothetical population of 10,000 impact craters formed under similar conditions (e.g., a similar target and impact parameters). That is, the measured distribution of radii from a single planform provides the best and only information concerning the distribution that is likely to be measured if a similar impact were repeated a large number of times.

Another significant measure of asymmetry is the planform aspect ratio or ellipticity, $a / b$, defined as the ratio of the major and minor axis lengths of an ellipse fitted to the planform. We have also measured the deviation from a fitted ellipse, to estimate the amount of radial deviation that is not mainly derived from an overall elliptical shape. This quantity is called the "residual deviation" $\left(\sigma_{E}\right)$ and is defined as the root mean square of the differences between individual radii of the planform and radii of an ellipse fitted to the planform. That is, if $R_{i}$ is the radius of the planform in the direction $\phi_{i}$ and $r_{i}$ is the radius of the fitted ellipse in the same direction, then $\sigma_{E} \equiv \sqrt{\frac{1}{N} \sum_{i}^{N}\left(R_{i}-r_{i}\right)^{2}}$. That is, $\sigma_{E}$ is a measure of the radial deviation after the contribution 


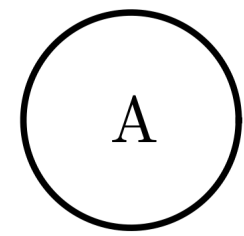

$\sigma_{R}=\sigma_{C}=\sigma_{E}=0.000$

$\bar{R}=1.000, \frac{a}{b}=1.00$

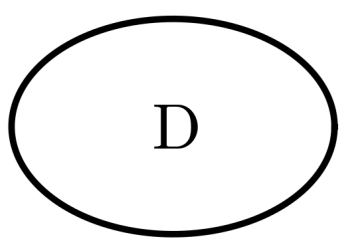

$\sigma_{R}=0.174, \frac{\sigma_{C}}{\sigma_{R}}=0.00$

$\sigma_{C}=0.000, \frac{\sigma_{E}}{\sigma_{R}}=0.00$

$\sigma_{E}=0.000, n_{0}=2$

$\bar{R}=1.212, \frac{a}{b}=1.50$

$\Omega=1.00$

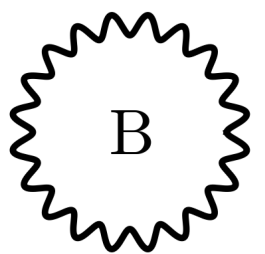

$\sigma_{R}=0.070, \frac{\sigma_{C}}{\sigma_{R}}=1.60$

$\sigma_{C}=0.113, \frac{\sigma_{E}}{\sigma_{R}}=1.02$

$\sigma_{E}=0.072, n_{0}=20$

$\bar{R}=1.000, \frac{a}{b}=1.00$

$\Omega=0.00$

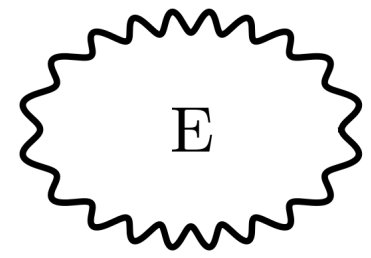

$\sigma_{R}=0.188, \frac{\sigma_{C}}{\sigma_{R}}=0.59$

$\sigma_{C}=0.110, \frac{\sigma_{E}}{\sigma_{R}}=0.38$

$\sigma_{E}=0.071, n_{0}=2$

$\bar{R}=1.212, \frac{a}{b}=1.49$

$\Omega=0.74$

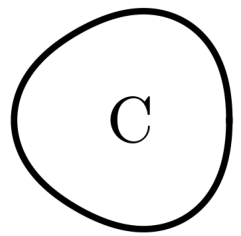

$\sigma_{R}=0.039, \frac{\sigma_{C}}{\sigma_{R}}=0.00$

$\sigma_{C}=0.000, \frac{\sigma_{E}}{\sigma_{R}}=1.00$

$\sigma_{E}=0.039, n_{0}=3$

$\bar{R}=1.000, \frac{a}{b}=1.00$

$\Omega=1.00$

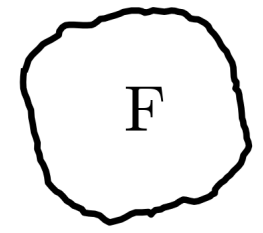

$$
\begin{gathered}
\sigma_{R}=0.083, \frac{\sigma_{C}}{\sigma_{R}}=0.19 \\
\sigma_{C}=0.016, \frac{\sigma_{E}}{\sigma_{R}}=0.56 \\
\sigma_{E}=0.046, n_{0}=4 \\
\bar{R}=1.000, \frac{a}{b}=1.06
\end{gathered}
$$$$
\Omega=0.67
$$

Figure 2: Morphometric parameters computed for example planforms. Part $\mathrm{F}$ is the rim planform of Barringer Crater. The parameters are defined in the text and Table 1. See text for discussion.

from an overall elongation is removed, and is in this sense "residual." To compare the residual deviation to the overall radial deviation, we compute $\sigma_{E} / \sigma_{R}$. When this value is close to 1 , the residual deviation accounts for all of the deviation from radial symmetry. When close to 0 , all the departure from symmetry is accounted for by an elliptical shape.

We also compute the "concave deviation," $\sigma_{C}$, defined as the root mean square of the radial difference between the planform and the convex hull. The convex hull is the smallest convex polygon that encompasses all of the points on the planform, and plays the same role in the definition of $\sigma_{C}$ as the fitted ellipse in the definition of $\sigma_{E}$. Finally, we also compare this deviation to the overall radial deviation as $\sigma_{C} / \sigma_{R}$. It is worth noting that whereas the maximum value of $\sigma_{E} / \sigma_{R}$ is $\approx 1, \sigma_{C} / \sigma_{R}$ can exceed 1 because the convex hull entirely bounds the planform. This is unlike the fitted ellipse, whose points occur inside as well as outside the planform. For this reason, concave deviation cannot be said to dominate the radial deviation unless $\sigma_{C} / \sigma_{R}>1$. The uncertainties for $\sigma_{C}$ as well as $\sigma_{E}$ were computed in the same fashion as the uncertainty of $\sigma_{R}$, 
using a bootstrap analysis.

The quantities described above are computed for example planforms in Figure 2. Part A shows a circle, for which the mean radius $\bar{R}$ is 1 ; there is zero radial deviation, so that $\sigma_{C}=\sigma_{E}=\sigma_{R}=$ 0 . A sine wave is added to this circle in part B. Since there is no overall elongation, it is still the case that $a / b=1$ and we note that $\sigma_{R} \approx \sigma_{E}$. A large amount of concave deviation has been introduced as well, causing $\sigma_{C}$ to become nonzero. Part $\mathrm{D}$ is an ellipse, in which all of the radial deviation derives from the overall elongation, so that $\sigma_{E}=0$. Part E shows the same ellipse with the earlier sine wave added. The residual deviation here is roughly the same as the overall radial deviation from part B: the deviation not caused by the elliptical shape. The concave deviation is also nearly identical with part B. The overall radial deviation, however, is larger than in parts B and $\mathrm{D}$ because both the elliptical component and the sine-wave component contribute to $\sigma_{R}$.

As mentioned, the second category of morphometric parameters were used to characterize planform shape as opposed to just the departure from symmetry. We used a Fourier decomposition of the planform following the approach in Eppler et al. (1983). This former study applied $\mathrm{R}$-factor analysis to identify linear combinations of circular harmonics that represent the most variation. Instead, we used the Fourier decomposition to assign planforms to shape categories. For each crater, the harmonic that contributes the most power for $n \geq 2$ (has the greatest amplitude) is called the "dominant harmonic" $\left(n_{0}\right)$ of the planform. The amplitudes $\left(\left|A_{n}\right|\right)$ and phase angles $\left(\phi_{n}\right)$ of terms in the Fourier series are implicitly defined by:

$$
R_{i}\left(\theta_{i}\right)=A_{0}+\sum_{n=1}^{360} A_{n} \cos \left(n \theta_{i}-\phi_{n}\right)
$$

An example is shown in Figure $2 \mathrm{~F}$ for the famously square-shaped planform of Barringer Crater (Shoemaker, 1960). Barringer Crater is classified as a square because its fourth harmonic amplitude is the strongest overall, so that the dominant harmonic $\left(n_{0}\right)$ is equal to 4 . Likewise, the dominant harmonic for the elliptical planforms in part $\mathrm{D}$ and $\mathrm{E}$ is $n_{0}=2$, whereas $n_{0}=3$ is computed from the tripolar shape in part $\mathrm{C}$. This enables us to characterize the distribution of crater shapes in terms of a histogram of dominant harmonics, as discussed later in Section 6. To quantify the contribution of a single harmonic to the overall deviation from symmetry, it is convenient to record the normalized amplitude $A_{n}^{*} \equiv\left|A_{n}\right| / \bar{R}$. We find that $A_{4}^{*} \approx 0.026$ for Barringer Crater. This amount of "quadrature" is extremely rare among well-preserved craters on Mars: we find that fewer than $2 \%$ of craters in HiRISE-simple exhibit a larger value of $A_{4}^{*}$.

Finally, we also define the "small harmonic fraction" $(\Omega)$ as the ratio of the sum of the harmonic amplitudes from 2 through 9 to the sum of the first 30 harmonics above 2 . We chose harmonics 2 through 9 because these are dominant in the case of at least one crater in our database. The maximum harmonic included in the denominator is determined by the resolution of the most coarsely-resolved planform in the data set. In the present case, all craters in our database are resolved by at least 32 harmonics.

$$
\Omega \equiv \frac{\sum_{n=2}^{9}\left|A_{n}\right|}{\sum_{n=2}^{32}\left|A_{n}\right|}
$$

The small harmonic fraction is a measure of the relative contribution of small harmonics in the deviation from symmetry. The radial deviation in planforms with low $\Omega$ is dominated by high-frequency (short-wavelength) deviations, whereas planforms with high $\Omega$ are dominated by low-frequency (long-wavelength) deviations. In this sense, high $\Omega$ suggests the planform has a strongly "polygonal" appearance. We estimate the uncertainty of $\Omega$ as the largest difference 
between harmonic amplitudes 8,9 , and 10 , divided by $\sum_{n=2}^{n=32}\left|A_{n}\right|$ (i.e., inclusion or omission of an additional low-order harmonic will modify $\Omega$ by at most this amount). In Figure $2, \Omega$ is 1.0 for shapes $\mathrm{C}$ and $\mathrm{D}$, which are entirely determined by low-order harmonics. The small harmonic fraction is undefined for a perfect circle (case A), and it is zero for a perfect circle with highfrequency sinusoidal variations (case B), because all the power is concentrated in a harmonic with $n>9$. The small harmonic fraction is intermediate between 0 and 1 for cases $\mathrm{E}$ through $\mathrm{F}$, in which the deviations from symmetry are distributed between low- and high-order harmonics.

Finally, we have also measured the orientation of the major axis of the fitted ellipse $\left(\theta_{E}\right)$ as well as the orientation of the second harmonic $\left(\phi_{2}\right)$. These quantities were measured in order to identify any systematic biases of the elongation direction, as may be caused by an impact angle bias such as found by Barlow (2015), or a bias from projection-related distortions. We found that the measured distributions of both quantities were indistinguishable from a uniform distribution for HiRISE-simple, LROC-complex, and HRSC-complex.

\section{Measurements of planform morphometry}

In this section we present the measurements of deviations from circular symmetry (radial deviation, concave deviation, residual deviation), and the small harmonic fraction. The other measures of planform shape and asymmetry (ellipticity and the dominant harmonics) are addressed in Section 6, where we will compare these measurements with calculations performed using the models developed in Section 5. The results in the present section are summarized in two tables. Table 2 contains the results of power-law fits to $\sigma_{R}$ vs. $D$ for HiRISE-simple, LROC-complex, and HRSC-complex. Table 3 summarizes statistically significant differences between subsets of our database, based on crater size and target strength. The target strength categories were defined in Section 2. Two threshold diameters were used to divide HiRISE-simple into a subset of small and large craters: $500 \mathrm{~m}$ and $1 \mathrm{~km}$. The latter diameter was chosen because previous work suggests that enhanced failure of the upper rim walls occurs for $D \geq 1 \mathrm{~km}$ in well-preserved martian simple craters (Watters et al., 2015). HRSC-complex and LROC-complex were divided approximately in half using the diameters of $10 \mathrm{~km}$ and $40 \mathrm{~km}$, respectively.

To obtain the results in Table 3, significant subset comparisons were identified by computing the Kolmogorov-Smirnov (KS) statistic and Anderson-Darling (AD) statistic for each pair of subsets. In Table 3, we only show comparisons that were significant at the $99 \%$ confidence level or above (i.e., $p$-value $\leq 0.01$ ) for $\mathrm{AD}$ or $\mathrm{KS}$ or both. For the overwhelming majority of results in this table, both statistics yielded $p \leq 0.01$ (i.e., if the two sets of measurements were drawn from the same distribution, the computed $\mathrm{KS}$ or $\mathrm{AD}$ statistic would occur by chance less than $1 \%$ of the time). For HiRISE-simple, we made comparisons based on the degree of modification, finding no significant differences for any of the parameters considered. The statistically significant comparisons are discussed in the following sections concerning simple craters and then complex craters, followed by terrain-dependent effects for both cases.

\subsection{Simple craters}

The radial deviation $\left(\sigma_{R}\right)$ of HiRISE-simple craters in the MM subset ("moderately modified") are shown in Figure 3A, spanning nearly two orders of magnitude in $\sigma_{R}$ as well as diameter $(D)$. These exhibit a power-law trend for $D \geq 100 \mathrm{~m}$, with a possible change in slope at small diameters $(D<100 \mathrm{~m})$. This transition is more visible in the box-and-whisker plots of the radius-normalized radial deviation $\left(\sigma_{R} / \bar{R}\right)$ shown in Figure 4. Part A of this figure shows $\sigma_{R} / \bar{R}$ 
measured from the rim planforms, while part B shows the same quantity measured from cavity planforms. In both cases, there is evidence of a change in slope at $D \approx 100 \mathrm{~m}$. That is, crater planforms become more symmetric (more circular) with increasing size for $D \geq 100 \mathrm{~m}$. Below this diameter, the size-dependence is absent or reversed. We suggest this transition relates to the depth of excavation, in which crater growth is influenced by variations in strength (Fulmer and Roberts, 1963), which for $D<100 \mathrm{~m}$ is less than $10 \mathrm{~m}$. That is, some small craters may form entirely in a relatively weak surface layer of sand or regolith where crater growth is not significantly influenced by strength variations, and for this reason will exhibit a relatively symmetrical shape at small sizes, lowering the average $\sigma_{R} / \bar{R}$ in this diameter range (creating the plateau for $D<100 \mathrm{~m}$ in Figure 4). This transition was also observed in earlier measurements derived from manual tracing of simple crater planforms in HiRISE imagery (Watters, 2009).

We fit a power law to the measured dependence of $\sigma_{R}$ vs. $D$ for many subsets of HiRISEsimple. The results of these fits are listed in Table 2. This includes results for a restricted range of diameters $(D \geq 100 \mathrm{~m})$, for craters that exhibit the best preservation, and for craters that formed in strong targets (lava plains and lava flows) and weak targets (crater floors, ejecta, debris, sediments). For rim planforms in the database as a whole, fitting a power law $\left(\sigma_{R}=a D^{m}\right)$ we found $m=0.745 \pm 0.015$. The exponent $m$ decreases for $D \geq 100 \mathrm{~m}$ to $m=0.674 \pm 0.019$. While maintaining this lower bound on the diameter, the exponent is even smaller for relatively unmodified craters $(m=0.649 \pm 0.022$ for "moderately modified" craters (MM) and $m=0.586 \pm$ 0.045 for "least modified" craters $(\mathrm{LM}))$ and for strong targets $(m=0.603 \pm 0.066)$. For the small number of relatively unmodified craters in strong targets with $D \geq 100 \mathrm{~m}, m=0.519 \pm 0.073$. In summary, smaller exponents were found for the HiRISE-simple rim planforms of craters with signs of better preservation and which formed in relatively strong targets. We found that this also holds for cavity planforms in HiRISE-simple (Table 2).

We also used the planform measurements to investigate ideas that derive from studies of the structure of well-preserved craters on Mars and the Earth. The structure of some simple craters, such as Endurance crater at Meridiani Planum on Mars and Barringer Crater near Winslow, AZ, USA, suggest that the transient cavity planform was highly concave (Watters et al., 2011). This conclusion derives from observing that the flap hinge is preserved in the corners or "vertices" of the planform but has been destroyed in between. If in some cases the transient planform is relatively unmodified by the rim collapse and slumping that characterizes the early modification stage of crater formation, then at least some well-preserved simple craters may exhibit concave planforms. We have plotted the cumulative distribution function (CDF) of the concave deviation for craters that are at most "moderately modified" (MM) in Figure 5. We find that planforms with $\sigma_{C} / \bar{R}$ comparable to the example in Figure 2B (i.e,. $\sigma_{C} / \bar{R}>0.10$ ) are nonexistent. Figure 5A shows that rim planforms exhibit a much higher degree of concavity when compared with cavity planforms. Concavities dominate the radial deviation $\left(\sigma_{C} / \sigma_{R}>1\right)$ in fewer than $20 \%$ of rim planforms and fewer than $5 \%$ of cavity planforms. We also find that $\sigma_{C} / \sigma_{R}$ is larger, on average, for rim planforms with $D<1 \mathrm{~km}$ (Table 3, rows 12-13). A similarly significant difference was not observed for cavity planforms.

We have also addressed a hypothesis proposed by Poelchau et al. (2009), that the flap hinge at Barringer Crater is especially uplifted in the corners of the square-shaped planform. By contrast, Watters et al. (2011) interpreted the same structures to imply that the hinge radius is simply much larger in the direction of Barringer's "diagonals", and that no excess uplift has occurred. If greater uplift commonly occurs in the "corners" of polygonal crater outlines, then we might expect to observe a correlation between planform radius $\left(R_{i}\right)$ measured at each rim position $i$ and the corresponding rim elevation $\left(h_{i}\right)$. We have measured Pearson's correlation $\rho_{R h}$ coefficient 

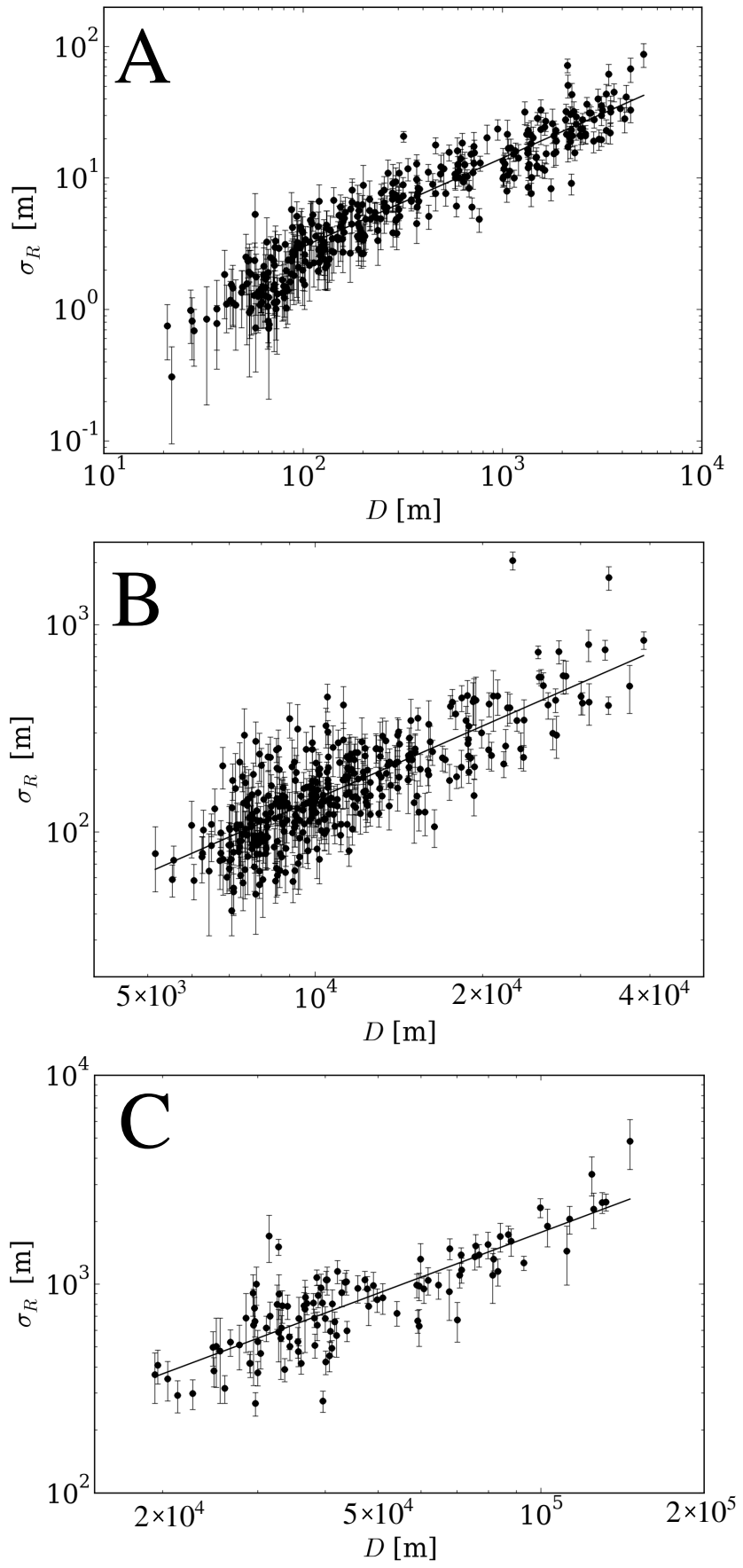

Figure 3: Radial deviation $\left(\sigma_{R}\right)$ of rim planforms versus log of crater diameter $(D)$ for: (A) martian simple craters in HiRISE-simple $(N=352)$; (B) martian complex craters in HRSC-complex $(N=382)$; (C) lunar complex craters in LROC-complex $(N=112)$. Solid lines are power-law fits spanning the entire range of diameters in B and $\mathrm{C}$, and only $D \geq 100 \mathrm{~m}$ in A. Fit parameters are reported in Table 2. The error bars were computed using the bootstrap method described in Section 3.3. 

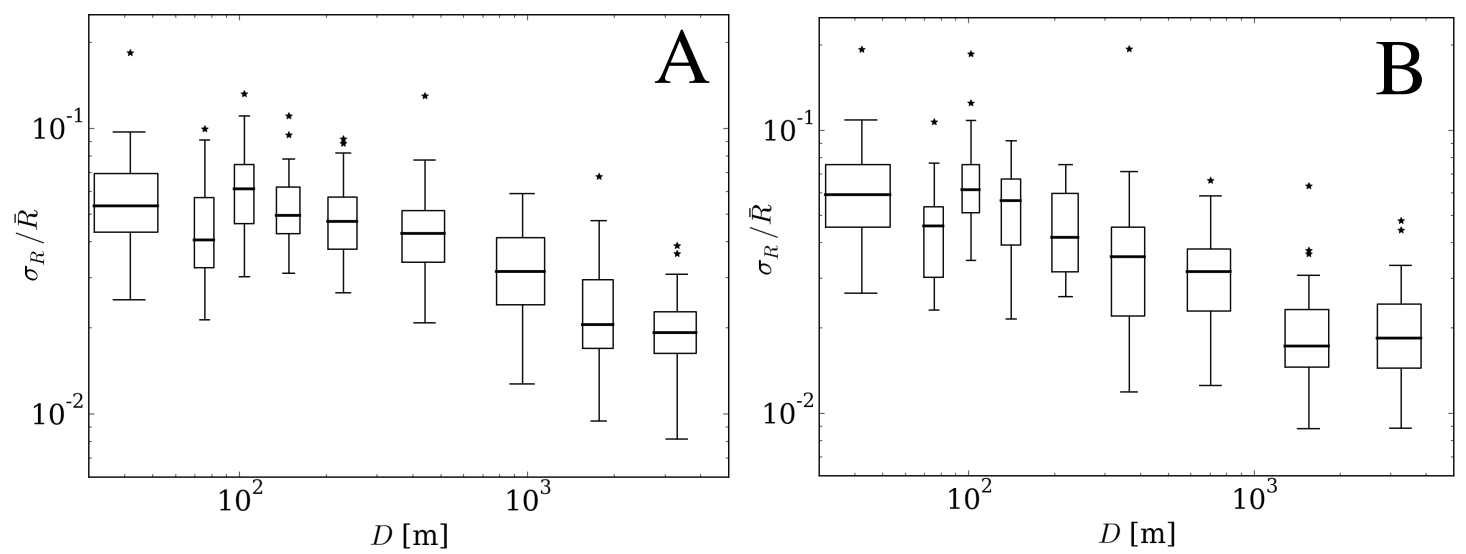

Figure 4: Box-whisker plots of the radius-normalized radial deviation of (A) rim planforms and (B) cavity planforms in HIRISE-simple. A change occurs at $D \approx 100 \mathrm{~m}$ : above this diameter, the radius-normalized radial deviation $\left(\sigma_{R} / \bar{R}\right)$ decreases. The plateau for $D<100 \mathrm{~m}$ may be the consequence of some small craters forming entirely in shallow, low-strength or strengthless layers, depressing the average in this diameter range. Whiskers extend to 1.5 times the interquartile range, and points represent outliers that occur beyond this range.
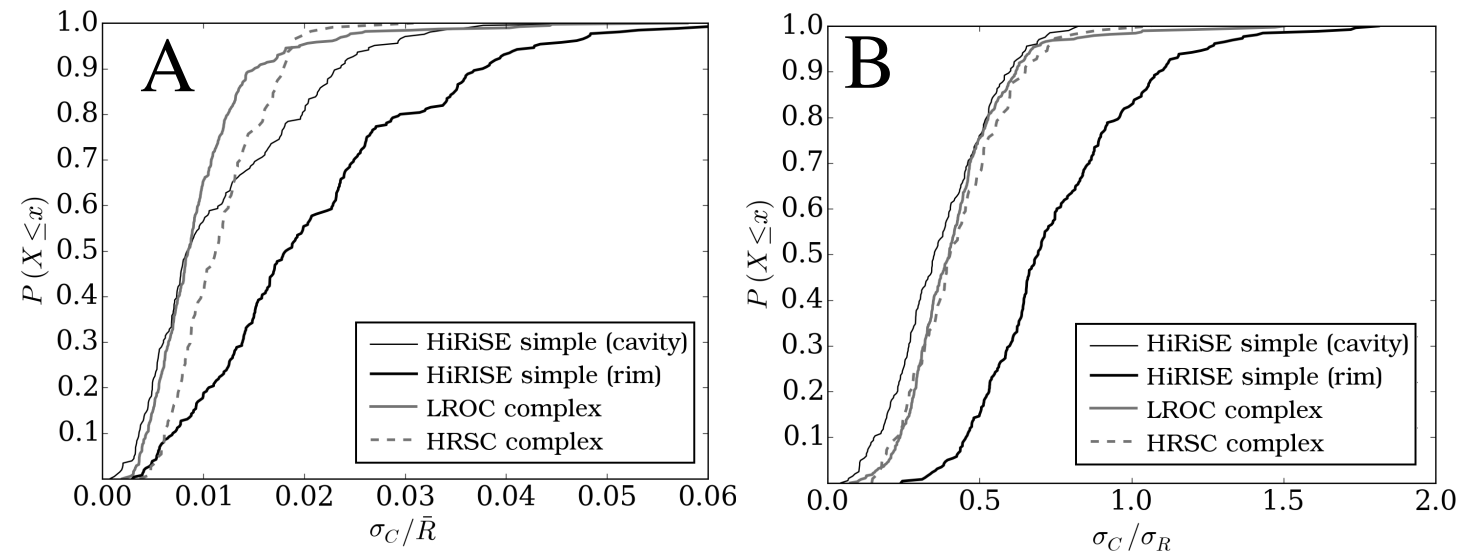

Figure 5: (A) Cumulative distribution function (CDF) for the radius-normalized concave deviation, $\sigma_{C} / \bar{R}$, for cavity planforms (thin black line) and rim planforms (thick black line) in HiRISE-simple/MM ("moderately modified", as defined in Watters et al. (2015)), as well as LROC-complex (gray line), and HRSC-complex (gray dashed line). (B) $\mathrm{CDF}$ for concave deviation as a fraction of overall radial deviation $\left(\sigma_{C} / \sigma_{R}\right)$. The rim planform of simple craters exhibit the highest degree of concavity overall; concave deviation dominates the radial deviation for a small fraction of rim planforms $(<20 \%)$ even in HiRISE-simple. 
for $R_{i}$ versus $h_{i}$ for craters in the MM subset of HiRISE-simple, finding that the distribution is symmetrical about zero. That is, we find no common tendency for a positive or negative correlation between rim radius and rim height: the corners of crater planforms are not elevated, in general, as one might expect if the flap hinge tends to deflect upward in "corners."

\subsection{Complex craters}

The radial deviations of crater planforms are plotted versus diameter for HRSC-complex in Figure 3B and LROC-complex in Figure 3C, along with power-law fits to these measurements. The fit parameters are reported in Table 2 . These measurements necessarily span only a limited range of diameters: slightly less than one order of magnitude. For this reason, the fitted power law parameters exhibit a relatively high degree of uncertainty. Unfortunately, this problem is insurmountable because the set of well-preserved complex craters on Mars and the Moon is small, and only span a small range of crater diameters. We have made these measurements on both worlds as a way of enhancing the relevance and confidence of any results found to be shared in common. We have also excluded proto-basins that occur at just two orders of magnitude in excess of the simple-complex transition diameters on both worlds (Baker et al., 2012).

We find the exponent $m \approx 1.0$ for the lunar complex craters in LROC-complex overall ( $m=$ $0.970 \pm 0.063$ ), ranging from $m=0.897 \pm 0.076$ for craters forming in the highlands ("weak" subset), to $m=1.05 \pm 0.113$ for craters forming in the maria ("strong" subset). This is consistent with prior results of Ronca and Salisbury (1966) and Murray and Guest (1970), who found no size dependence of the departure from symmetry as measured using different morphometric parameters. Like Ronca and Salisbury (1966), Baker and Head (2013) and Pike (1977) used a circularity index but instead found a decrease in circularity with increasing diameter for complex craters on the Moon. We suggest that the discrepancy may have several possible sources. First, it is possible that some transitional forms were included in the dataset (as is necessarily true for Pike (1977)), which will have a planform shape intermediate between true complex craters and the highly symmetric, large-diameter simple craters. A second consideration is that circularity is not just sensitive to the scale of radial departures from symmetry; the total perimeter length, sinuosity, and fractal dimension of the planform can also influence this quantity. We separately addressed some of these aspects of the diameter dependence using the "small harmonic fraction," defined in Section 3.3, which exhibits a strong diameter dependence for lunar complex craters (see below). Baker and Head (2013) found no dependence of circularity on diameter for complex craters on Mercury.

For the martian complex craters in HRSC-complex, we found that $m$ somewhat exceeds 1 overall $(m=1.173 \pm 0.048)$. A similar value of the exponent was calculated for craters in weak targets $(m=1.182 \pm 0.056)$ and a smaller value for strong targets $(m=1.044 \pm 0.100)$. To evaluate the influence of extreme outliers on our results, we removed the two outliers that occur far above the trend in the upper-right corner of Figure 3B, finding that the exponent decreases somewhat to $m=1.131 \pm 0.047$ for HRSC-complex as a whole. We also recomputed our results after restoring the planforms to the projection of the original HRSC orthoimages. On average, this had the effect of changing $\sigma_{R}$ by $1.75 \%$, with a $<1 \%$ change in the value of $m$. In summary, the deviations from circular symmetry scale approximately linearly with the diameter of complex craters, and may be slightly nonlinear with $m>1$ on Mars. In addition to prior work mentioned earlier, this is consistent with the results of an early study that found no strong diameter dependence of radial deviation for martian complex craters (Oberbeck et al., 1972).

The concave deviation was also computed for complex craters; the cumulative distribution functions of this quantity for HRSC-complex and LROC-complex are shown in Figure 5. The 
Table 2: Power law fits to radial deviation as a function of diameter: $\sigma_{R}=a D^{m}$ where $m$ and $a$ are the reported fit parameters. "F" refers to a database filter; "*” implies only craters with $D \geq 100$ m were included. Subset abbreviations are defined as follows: HS = HiRISE-simple; LC = LROC-complex; HC = HRSC-complex; rim = rim planform; cav = cavity planform; $\mathrm{MM}=$ moderately modified; $\mathrm{LM}=$ least modified; strong $\mathrm{I}=$ lava flow and plains units mapped by Tanaka et al. (2014); weak I = complement of strong I; strong II = complement of weak II; weak II = geologic units dominated by sediments and crater-related units as mapped in Tanaka et al. (2014) and supplemented with HiRISE observations. Also listed are values of Pearson's correlation coefficient $(\rho)$ and the number of planforms $(N)$.

\begin{tabular}{|c|c|c|c|c|c|c|}
\hline No. & $\mathrm{F}$ & Subset & $m$ & $a$ & $N$ & $\rho$ \\
\hline 1 & & HS/rim: all & $0.745 \pm 0.015$ & $0.082 \pm 0.007$ & 352 & 0.94 \\
\hline 2 & & HS/rim: MM & $0.736 \pm 0.017$ & $0.088 \pm 0.009$ & 261 & 0.94 \\
\hline 3 & & HS/rim: LM & $0.616 \pm 0.035$ & $0.193 \pm 0.044$ & 56 & 0.92 \\
\hline 4 & & HS/cav: all & $0.701 \pm 0.018$ & $0.094 \pm 0.009$ & 299 & 0.91 \\
\hline 5 & & HS/cav: MM & $0.699 \pm 0.021$ & $0.095 \pm 0.011$ & 205 & 0.92 \\
\hline 6 & & HS/cav: LM & $0.614 \pm 0.056$ & $0.164 \pm 0.057$ & 38 & 0.88 \\
\hline 7 & * & HS/rim: all & $0.674 \pm 0.019$ & $0.134 \pm 0.017$ & 251 & 0.91 \\
\hline 8 & $*$ & HS/rim: MM & $0.649 \pm 0.022$ & $0.161 \pm 0.023$ & 191 & 0.91 \\
\hline 9 & * & HS/rim: LM & $0.586 \pm 0.045$ & $0.239 \pm 0.073$ & 49 & 0.88 \\
\hline 10 & * & HS/cav: all & $0.651 \pm 0.025$ & $0.131 \pm 0.020$ & 212 & 0.87 \\
\hline 11 & * & HS/cav: MM & $0.643 \pm 0.029$ & $0.139 \pm 0.026$ & 145 & 0.88 \\
\hline 12 & * & HS/cav: LM & $0.597 \pm 0.072$ & $0.183 \pm 0.087$ & 32 & 0.84 \\
\hline 13 & * & HS/rim: all & $0.674 \pm 0.019$ & $0.134 \pm 0.017$ & 251 & 0.91 \\
\hline 14 & $*$ & HS/rim: weak I & $0.687 \pm 0.019$ & $0.122 \pm 0.015$ & 205 & 0.93 \\
\hline 15 & * & HS/rim: strong I & $0.603 \pm 0.066$ & $0.220 \pm 0.093$ & 46 & 0.81 \\
\hline 16 & $*$ & HS/rim: weak II & $0.797 \pm 0.042$ & $0.064 \pm 0.016$ & 46 & 0.94 \\
\hline 17 & * & HS/rim: strong II & $0.645 \pm 0.022$ & $0.162 \pm 0.023$ & 205 & 0.90 \\
\hline 18 & * & HS/cav: all & $0.681 \pm 0.026$ & $0.098 \pm 0.016$ & 212 & 0.88 \\
\hline 19 & * & HS/cav: weak I & $0.695 \pm 0.026$ & $0.091 \pm 0.015$ & 175 & 0.90 \\
\hline 20 & * & HS/cav: strong I & $0.565 \pm 0.093$ & $0.187 \pm 0.105$ & 37 & 0.72 \\
\hline 21 & * & HS/cav: weak II & $0.803 \pm 0.057$ & $0.054 \pm 0.019$ & 41 & 0.91 \\
\hline 22 & * & HS/cav: strong II & $0.656 \pm 0.029$ & $0.112 \pm 0.020$ & 171 & 0.87 \\
\hline 23 & * & HS/rim: weak I, MM & $0.670 \pm 0.021$ & $0.137 \pm 0.019$ & 156 & 0.93 \\
\hline 24 & * & HS/rim: strong I, MM & $0.519 \pm 0.073$ & $0.417 \pm 0.201$ & 35 & 0.78 \\
\hline 25 & * & HS/rim: weak II, MM & $0.806 \pm 0.053$ & $0.064 \pm 0.021$ & 32 & 0.94 \\
\hline 26 & * & HS/rim: strong II, MM & $0.619 \pm 0.023$ & $0.193 \pm 0.030$ & 159 & 0.90 \\
\hline 27 & $*$ & HS/cav: all, MM & $0.675 \pm 0.030$ & $0.103 \pm 0.020$ & 145 & 0.88 \\
\hline 28 & $*$ & HS/cav: weak I, MM & $0.694 \pm 0.030$ & $0.091 \pm 0.018$ & 123 & 0.90 \\
\hline 29 & $*$ & $\mathrm{HS} /$ cav: strong I, MM & $0.523 \pm 0.113$ & $0.270 \pm 0.189$ & 22 & 0.72 \\
\hline 30 & $*$ & HS/cav: weak II, MM & $0.858 \pm 0.070$ & $0.039 \pm 0.017$ & 28 & 0.92 \\
\hline 31 & $*$ & HS/cav: strong II, MM & $0.635 \pm 0.031$ & $0.128 \pm 0.026$ & 117 & 0.88 \\
\hline 32 & & LC: all & $0.970 \pm 0.063$ & $0.025 \pm 0.017$ & 112 & 0.83 \\
\hline 33 & & LC: weak & $0.897 \pm 0.076$ & $0.057 \pm 0.047$ & 84 & 0.79 \\
\hline 34 & & LC: strong & $1.050 \pm 0.113$ & $0.010 \pm 0.011$ & 28 & 0.88 \\
\hline 35 & & HC: all & $1.174 \pm 0.048$ & $0.0029 \pm 0.0013$ & 382 & 0.78 \\
\hline 36 & & HC: weak I & $1.186 \pm 0.056$ & $0.0027 \pm 0.0014$ & 280 & 0.79 \\
\hline 37 & & HC: strong I & $1.042 \pm 0.101$ & $0.0090 \pm 0.0084$ & 102 & 0.72 \\
\hline
\end{tabular}


Table 3: Statistically significant comparisons of morphometric parameter values accomplished using the KolmogorovSmirnov (KS) and Anderson-Darling (AD) tests; in all cases, differences are significant at the $99 \%$ confidence level or greater. Compared subsets are based upon target strength (rows 1-11) and crater diameter (rows 12-23). Symbols representing morphometric quantities are defined in Table 1. Subset abbreviations are defined as follows: HS = HiRISEsimple; LC = LROC-complex; HC = HRSC-complex; rim = rim planform; cav = cavity planform; $\mathrm{MM}=$ moderately modified; LM = least modified; strong I = lava flow and lava plains units mapped by Tanaka et al. (2014); weak I = complement of strong I; strong II = complement of weak II; weak II = geologic units dominated by sediments and craterrelated units as mapped in Tanaka et al. (2014) and supplemented with HiRISE observations. "F" refers to a database filter; "†" implies that only craters in MM were included, while " $\ddagger$ " implies craters in MM with $D \geq 100 \mathrm{~m}$. Note that $N_{X}$ is the number in subset $X$, while $q_{X} \pm \sigma_{X}$ is the mean and standard deviation of the quantity (q) for subset $X$ (where $X$ is $\mathrm{A}$ or $\mathrm{B})$.

\begin{tabular}{|c|c|c|c|c|c|c|c|c|}
\hline No. & $q$ & $\mathrm{~F}$ & Subset A & $N_{A}$ & Subset B & $N_{B}$ & $q_{A} \pm \sigma_{A}$ & $q_{B} \pm \sigma_{B}$ \\
\hline 1 & $\sigma_{C} / \bar{R}$ & & LC: weak & 84 & LC: strong I & 28 & $0.0161 \pm 0.0060$ & $0.0129 \pm 0.0052$ \\
\hline 2 & $\sigma_{C} / \bar{R}$ & & HC: weak I & 282 & HC: strong I & 103 & $0.0137 \pm 0.0076$ & $0.0103 \pm 0.0076$ \\
\hline 3 & $\sigma_{E} / \bar{R}$ & & LC: weak & 84 & LC: strong I & 28 & $0.0316 \pm 0.0079$ & $0.0269 \pm 0.0068$ \\
\hline 4 & $\sigma_{E} / \bar{R}$ & & HC: weak I & 282 & HC: strong I & 103 & $0.0257 \pm 0.0087$ & $0.0227 \pm 0.0071$ \\
\hline 5 & $\sigma_{C} / \sigma_{R}$ & & HC: weak I & 282 & HC: strong I & 103 & $0.437 \pm 0.174$ & $0.367 \pm 0.192$ \\
\hline 6 & $\sigma_{C} / \sigma_{R}$ & & HS/cav: weak II & 72 & HS/cav: strong II & 227 & $0.317 \pm 0.154$ & $0.384 \pm 0.166$ \\
\hline 7 & $\sigma_{C} / \sigma_{R}$ & $\ddagger$ & HS/cav: weak II & 52 & HS/cav: strong II & 153 & $0.295 \pm 0.147$ & $0.397 \pm 0.165$ \\
\hline 8 & $\sigma_{C} / \sigma_{R}$ & $\dagger$ & HS/cav: weak II & 28 & HS/cav: strong II & 117 & $0.327 \pm 0.136$ & $0.412 \pm 0.165$ \\
\hline 9 & $\sigma_{E} / \sigma_{R}$ & $\ddagger$ & HS/cav: weak II & 52 & HS/cav: strong II & 153 & $0.684 \pm 0.188$ & $0.787 \pm 0.154$ \\
\hline 10 & $\Omega$ & $\ddagger$ & HS/cav: weak II & 52 & HS/cav: strong II & 153 & $0.716 \pm 0.068$ & $0.673 \pm 0.087$ \\
\hline 11 & $\Omega$ & & HC: weak I & 282 & HC: strong I & 103 & $0.633 \pm 0.073$ & $0.660 \pm 0.074$ \\
\hline 12 & $\overline{\overline{\sigma_{C} / \sigma_{R}}}$ & & " HS/rim: $D<1 \mathrm{~km}$ & 256 & "HS/rim: $D \geq 1 \mathrm{~km}$ & 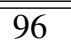 & 0.769 \pm 0.239 & $0.728 \pm 0.294$ \\
\hline 13 & $\sigma_{C} / \sigma_{R}$ & $\dagger$ & HS/rim: $D<1 \mathrm{~km}$ & 105 & $\mathrm{HS} /$ rim: $D \geq 1 \mathrm{~km}$ & 86 & $0.811 \pm 0.272$ & $0.726 \pm 0.298$ \\
\hline 14 & $\sigma_{C} / \sigma_{R}$ & & LC: $D<40 \mathrm{~km}$ & 56 & LC: $D \geq 40 \mathrm{~km}$ & 56 & $0.336 \pm 0.112$ & $0.518 \pm 0.171$ \\
\hline 15 & $\sigma_{C} / \sigma_{R}$ & & $\mathrm{HC}: D<10 \mathrm{~km}$ & 187 & $\mathrm{HC}: D \geq 10 \mathrm{~km}$ & 198 & $0.359 \pm 0.144$ & $0.475 \pm 0.196$ \\
\hline 16 & $\sigma_{E} / \sigma_{R}$ & & $\mathrm{HC}: D<10 \mathrm{~km}$ & 187 & $\mathrm{HC}: D \geq 10 \mathrm{~km}$ & 198 & $0.811 \pm 0.120$ & $0.839 \pm 0.133$ \\
\hline 17 & $\Omega$ & & HS/cav: $D<1 \mathrm{~km}$ & 231 & HS/cav: $D \geq 1 \mathrm{~km}$ & 68 & $0.699 \pm 0.077$ & $0.647 \pm 0.097$ \\
\hline 18 & $\Omega$ & $\dagger$ & HS/cav: $D<1 \mathrm{~km}$ & 147 & HS/cav: $D \geq 1 \mathrm{~km}$ & 58 & $0.700 \pm 0.077$ & $0.644 \pm 0.091$ \\
\hline 19 & $\Omega$ & & HS/cav: $D<500 \mathrm{~m}$ & 202 & HS/cav: $D \geq 500 \mathrm{~m}$ & 97 & $0.705 \pm 0.075$ & $0.650 \pm 0.092$ \\
\hline 20 & $\Omega$ & $\dagger$ & HS/cav: $D<500 \mathrm{~m}$ & 129 & HS/cav: $D \geq 500 \mathrm{~m}$ & 76 & $0.706 \pm 0.076$ & $0.646 \pm 0.087$ \\
\hline 21 & $\Omega$ & $\ddagger$ & HS/cav: $D<500 \mathrm{~m}$ & 69 & $\mathrm{HS} / \mathrm{cav}: D \geq 500 \mathrm{~m}$ & 76 & $0.684 \pm 0.073$ & $0.646 \pm 0.087$ \\
\hline 22 & $\Omega$ & & LC: $D<40 \mathrm{~km}$ & 56 & LC: $D \geq 40 \mathrm{~km}$ & 56 & $0.700 \pm 0.070$ & $0.607 \pm 0.084$ \\
\hline 23 & $\Omega$ & & HC: $D<10 \mathrm{~km}$ & 187 & HC: $D \geq 10 \mathrm{~km}$ & 198 & $0.661 \pm 0.073$ & $0.621 \pm 0.070$ \\
\hline
\end{tabular}



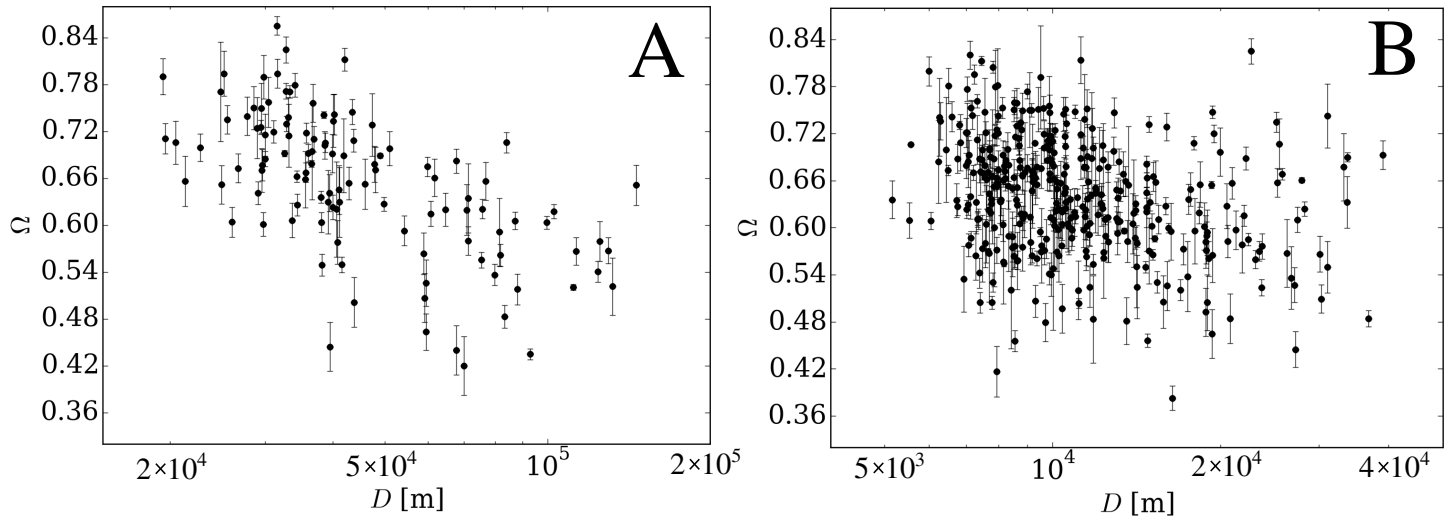

Figure 6: Small harmonic fraction $(\Omega)$ as a function of log diameter for (A) LROC-complex, showing a marked decrease with increasing crater size (Pearson correlation coefficient $\rho=-0.56$ ). (B) A similarly strong negative correlation is not observed for complex craters on Mars $(\rho=-0.25)$. Nonetheless, small complex craters on Mars $(D<10 \mathrm{~km})$ exhibit a larger $\Omega$, on average, when compared with large complex craters $(D>10 \mathrm{~km})$; see Table 3, row 23.

distributions of concave fraction $\left(\sigma_{C} / \sigma_{R}\right)$ are essentially identical for lunar and martian complex craters and cavity planforms in HiRISE-simple (Figure 5B). Normalized by average radius, lunar complex craters exhibit the least overall concave deviation (Figure 5A).

As a fraction of the overall radial deviation, the concave deviation of small complex craters on Mars $(D<10 \mathrm{~km})$ and the Moon $(D<40 \mathrm{~km})$ is less than measured for larger diameters (Table 3 , rows 14-15). That is, concavities comprise a more significant fraction of the deviation from symmetry at large sizes for complex craters on both worlds. The elliptical deviation changes far less with size among complex craters; a small but statistically significant difference is measured for craters on Mars when comparing $D<10 \mathrm{~km}$ and $D \geq 10 \mathrm{~km}$ (Table 3, row 16).

The small harmonic fraction $(\Omega)$ for craters in LROC-complex is plotted in Figure 6A and shows a clear negative trend with increasing crater diameter. That is, low-order harmonics contribute more to the deviation from symmetry for small complex craters versus larger ones, consistent with the results of Eppler et al. (1983). It is worth noting that Pohn and Offield (1970) qualitatively identified a stronger "polygonality" of lunar complex craters below $48 \mathrm{~km}$ in diameter. We note that $\Omega>0.72$ only for $D<50 \mathrm{~km}$, and all craters with $\Omega<0.54$ are $\sim 40$ $\mathrm{km}$ in diameter or larger. Row 23 in Table 3 shows that $\Omega$ is also larger, on average, for small martian complex craters $(D<10 \mathrm{~km})$, although the dependence is less pronounced (Figure 6B). On average, the small harmonic fraction also decreases with increasing diameter for cavity planforms in HiRISE-simple. No significant size dependence of $\Omega$ was observed for rim planforms in HiRISE-simple.

\subsection{Terrain dependence}

We have already noted the influence of target strength on the diameter scaling of radial deviation in simple craters on Mars. Using two different ways of classifying targets into strong vs. weak (Section 2), the scaling exponent $m$ is smaller in stronger targets for cavity and rim planforms alike (Table 2, rows 14-31). On Mars and the Moon, the diameter scaling of radial deviation was measured to be $m \approx 1.04-1.05$ for strong targets, but was smaller for weak targets 
on the Moon and larger for weak targets on Mars (Table 2, rows 32-37). As cautioned earlier, however, these fit parameters contain large uncertainties of nearly $10 \%$ owing to the relatively small range of diameters over which measurements are possible.

Results concerning the influence of terrain type on the distribution of concave and elliptical deviation, as well as the small harmonic fraction, are reported in Table 3. Among complex craters on the Moon as well as Mars, the radius-normalized residual and concave deviations $\left(\sigma_{E} / \bar{R}\right.$ and $\sigma_{C} / \bar{R}$, respectively) are larger, on average, for craters that formed in weaker targets (Table 3, rows 1-4). We also find that the concave deviation comprises a much larger fraction of the overall radial deviation among martian complex craters in relatively weak targets (Table 3, row 5). The reason is not known; we suggest this may relate to the way terraces form in complex craters where targets have deep versus shallow megaregoliths. In the former case, wall collapse may exhibit a tendency to form localized, scalloped terraces, versus what happens in competent rock with a greater tendency to form long, straight wall segments along through-going faults. At least among martian complex craters, crater planforms in weak targets are shaped more strongly by high-order (high frequency) harmonics (Table 3, row 11).

For craters in HiRISE-simple, the radial deviation of cavity planforms is more strongly dominated by concavity in stronger targets (Table 3, rows 6-8). This observation is consistent with the idea that concavities occur in targets with strength heterogeneities in which the excavation flow exploits strength anisotropies, creating transiently concave cavities. For example, the hinge trace of the transient cavity that formed during the excavation of Barringer Crater on Earth as well as Endurance crater at Meridiani Planum on Mars were found to have a concave planform (Watters et al., 2011). It is important to note, however, that the result in Table 3 was only observed for cavity planforms, and using just one of two methods for distinguishing strong and weak targets. The fraction of the radial deviation dominated by deviations from an ellipse is also larger, on average, for HiRISE-simple cavity planforms in strong targets (Table 3, row 9).

Finally, row 10 of Table 3 suggests that cavity planforms are somewhat more dominated by low-order harmonics in weak targets. Put another way, deviations from circular symmetry in strong targets are higher-frequency (they comprise, on average, a smaller fraction of the circumference) than what occurs in weak targets. The reason for this is not known, although the reason may lie with the greater strength variations expected in stronger targets. We speculate that the extra "raggedness" of the cavity planform results from small-scale variations in strength exploited by the excavation flow in the final stages of crater growth.

\section{Scaling arguments and geometrical models}

Three-dimensional hydrocode models involving realistic strength heterogeneities are computationally expensive. The time required to compute a large number of simulations, in order to estimate the statistical variation observed in nature, is computationally prohibitive. We therefore turn to simple scaling arguments and geometrical models to shed light on the measured distribution of planform shapes and departure from symmetry. Such models can illuminate the extent to which measured relationships derive from simple geometrical and statistical principles. In this section, we develop scaling arguments which can be used to motivate expectations about the dependence of radial deviation upon crater size, as well as the distribution of crater shapes. As mentioned, we are less concerned with the elongation asymmetry created by highly oblique impacts, expected to influence only a small fraction of all craters (Bottke et al., 2000; Collins et al., 2011), and will instead focus on the influence of heterogeneities in strength. 
We examine two categories of model planforms, which are constructed from prescribed distributions of defect sizes in target materials. In particular, we construct planforms by (i) connecting line segments of varying length in a quasi-symmetric arrangement by "segmenting" a circle; and (ii) connecting the end points of a fixed number of rays of variable length, which emanate from a common origin. The first two models that we examine belong to category (i) and involve replacing arcs of a circle with straight line segments. The third model is of the second type (ii), and is motivated by physical arguments regarding how strength flaws influence the excavation flow to produce asymmetries. All three models generate polygonal planforms, which may be considered a low-order approximation of rim planforms in nature.

\subsection{Segmented rim model: fixed defect size}

In the simplest model, we assume that the length scale of deviations from planimetric symmetry is similar to the length scale of strength heterogeneities or defects in the target. In the following discussion, we use the symbol $\bar{\lambda}$ to represent the characteristic or limiting size of strength heterogeneities. For example, $\bar{\lambda}$ may represent the average, modal, or maximum length of deep fractures in a heavily-fractured target, or the average or maximum separation between them.

The model is illustrated in Figure 7A, where we have shown equilateral polygons with a constant side length, $\ell$, in this case equal to the length scale of the strength heterogeneities, $\bar{\lambda}$. Also shown is an inscribed circle in gray. In physical terms, this model represents one of the following scenarios: (a) in the final stages of crater growth, the excavation encounters resistance from strength heterogeneities with characteristic size $\bar{\lambda}$; or (b) a symmetrical crater is excavated (shown in gray) and the early modification causes slumping that results in a polygon whose edges have the length $\bar{\lambda}$ of preexisting target fractures.

The maximum radial deviation $\Delta R$ (the maximum distance between the gray circle of radius $R$ and the polygon in which it is inscribed) is given by:

$$
\Delta R=R\left(1-\sqrt{1-\left(\frac{\ell}{2 R}\right)^{2}}\right) \approx \frac{\ell^{2}}{8 R} \text { for } \ell / 2 R \ll 1
$$

Since $\ell$ is a constant, this relation shows that the maximum radial deviation depends on the inverse of the radius, and hence also the inverse of the diameter $\left(\Delta R \sim D^{-1}\right)$. Because the average difference between the radius of the inscribed polygon and circle are obviously proportional to this maximum difference, it follows that the radial deviation $\sigma_{R}$ will also depend on the planform diameter as $\sigma_{R} \sim D^{m}$ where $m \approx-1$.

As already discussed in the previous section, real impact craters exhibit a power-law size scaling of radial deviation with a positive exponent, ranging between 0.5 and 0.75 for simple craters and $m \approx 1.0$ for complex craters. It is therefore clear that measured deviations from symmetry are not of a fixed size, and do not merely correspond to the scale of fixed-size defects in the target. We turn next to a segmented rim model with defect size that depends on crater size.

\subsection{Segmented rim model: variable defect size}

We now address the more general case in which the polygon side or segment length $(\ell)$ is selected from a distribution whose average is a simple function of the radius $R$. As a result, the diameter dependence of $\sigma_{R}$ is effectively prescribed in advance. The planforms are again 


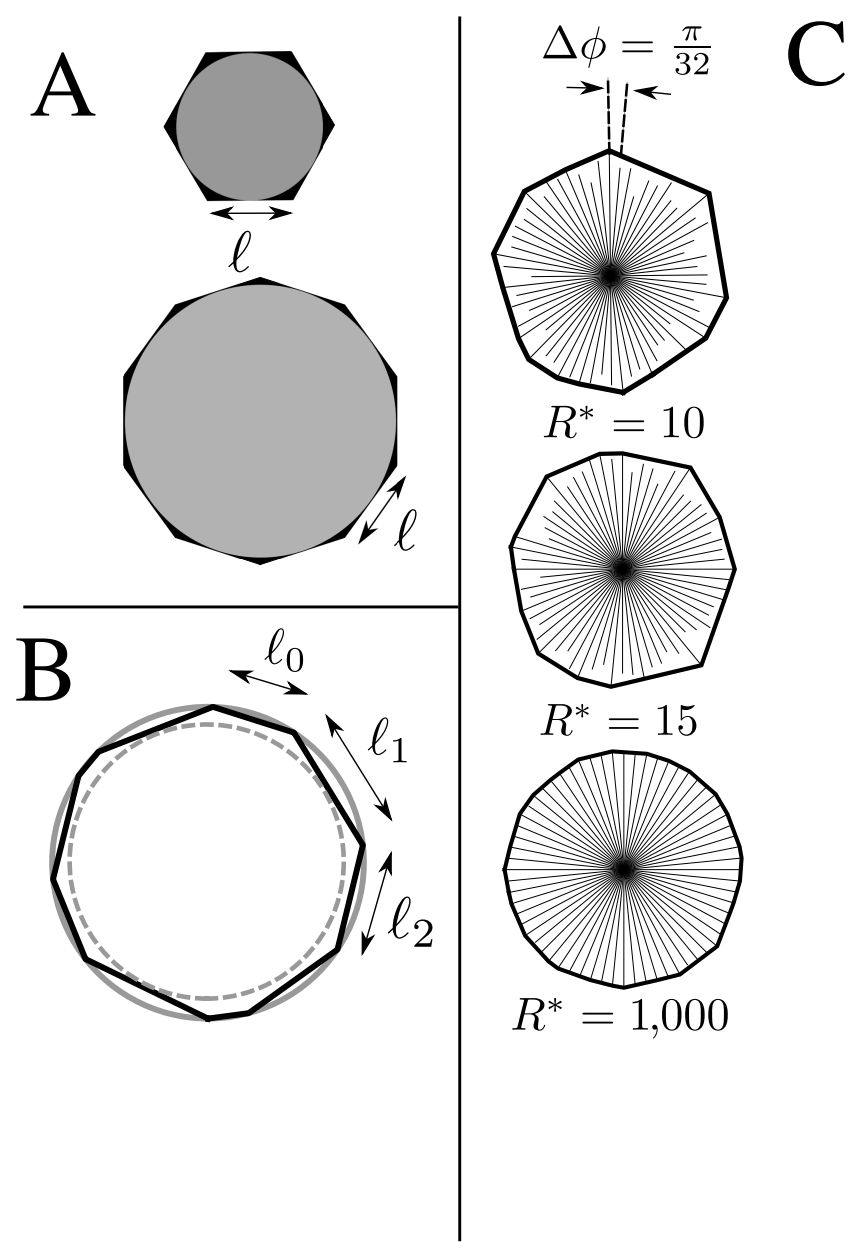

Figure 7: Synthetic planforms generated using simple geometrical models. (A) Equilateral polygons with constant edge length $\ell$ equal to the characteristic size of strength flaws. In this case, $\sigma_{R} \sim D^{m}$ where $m=-1$. (B) Illustration of the "Segmentation of Circular Rim" model (SOCR), in which circular arcs are replaced with chords whose lengths are chosen from a uniform or normal distribution. In this case, the scaling is prescribed by the model (e.g., $m \equiv 1$ ). (C) Example planforms constructed using the "Convex Hull of Radial Sum" model (CHORS), for which $m \approx 0.6$ is an intrinsic property. Note that $R^{*} \equiv \bar{R} / \bar{\lambda}$ and $\Delta \phi$ is the angular separation between model "rays" along which the radial sums are computed. 
constructed by connecting line segments. In this case, these are sampled from an assumed distribution of segment lengths. The purpose of this model is to create a statistical realization of synthetic planforms whose diameter scaling for $\sigma_{R}$ is prescribed to agree with measurements, and then to compare the synthetic planforms with the observed distribution of dominant harmonics.

As shown in Section $4, \sigma_{R} \approx a D^{m}$ where $m$ is roughly equal to or somewhat greater than 1 for complex craters. Early studies found that the overall deviation from symmetry does not change with the size of complex craters (Ronca and Salisbury, 1966; Murray and Guest, 1970), consistent with $m \approx 1$. Recent work has described the tendency for basin-sized features to be more elliptical, on average, consistent with $m>1$ in this limit (Andrews-Hanna and Zuber, 2010), although for reasons that our models cannot address. We consider the simple case in which the average model segment length is a constant fraction of the radius: $\bar{\ell} \equiv k R$ for constant $k$; we leave the examination of a nonlinear dependence for future work.

We proceed by constructing a statistical realization of the polygonal planforms described earlier, hereafter called the "Segmentation of Circular Rim" model (SOCR). An example planform is shown in Figure 7B. The construction of a SOCR planform begins with sampling a sequence of segment lengths $\ell_{0}, \ell_{1}, \ldots \ell_{j}$ from a distribution characterized by a prescribed mean $(\bar{\ell} \equiv k R)$ and width.

To the extent that segments of real crater planforms are approximately straight, they exhibit no minimum size. We therefore ensure that segment length can be arbitrarily small by choosing distribution widths that encompass $\ell=0$. For example, in the case of a uniform distribution, the range $\Delta \ell$ of the $\ell_{j}$ is set equal to $2 \bar{\ell}$ so that segment lengths can range in size down to zero meters. For the case of a normal distribution, the standard deviation is set equal to that of the uniform distribution in which $\Delta \ell=2 \bar{\ell}$.

SOCR planforms are constructed as follows: while tracing a circle, line segment lengths are sampled from a uniform or normal distribution and added to the polygon, connecting vertices until the current segment length exceeds the distance between the last point and the first. These two points are then joined by a final bridging segment. That is, at least one side of the polygonal model planform is never directly sampled from the segment length distribution. This final segment length is, however, guaranteed to be encompassed by the range of selectable values provided $\Delta \ell=2 \bar{\ell}$, as mentioned. All planform vertices lie on the circle, which was "segmented" in the planform construction process. We save for Section 6 a discussion of morphometric parameters computed from SOCR model planforms.

The goal of the SOCR model is to construct artificial planforms by connecting segments whose lengths are sampled from a statistical distribution, such as might be expected if a symmetrical cavity with a circular planform is modified in a collapse process that is influenced by defects with an identical or similar size distribution. As will be discussed in Section 6, model planforms constructed in this way, using a symmetric distribution for the $\ell_{j}$, such as a uniform or normal distribution, are a poor match for the observed distribution of dominant harmonics. Future work might usefully examine whether an asymmetric distribution, such a power law, produces a more realistic distribution of planform shapes.

\subsection{Radial sum model: fixed defect size}

As we saw earlier, $\sigma_{R} \sim D^{m}$ for $m$ ranging from 0.5 to 0.65 for strong targets and relatively well-preserved craters. This is similar to $m=1 / 2$, which results from scaling in random walks and diffusive processes, a consequence of summing random numbers. That $m>1 / 2$ instead of $m=1 / 2$ may be the result of correlations or pseudo-randomness, a possibility that we explore in 


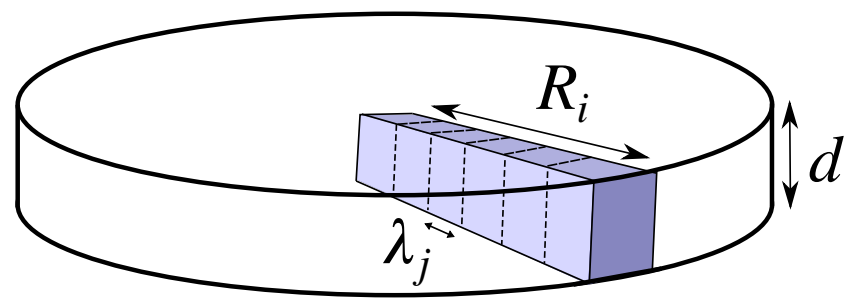

Figure 8: Illustration of a slice $i$ in the radial sum model. The slice is made up of discrete elements which represent strength heterogeneities of variable size $\lambda_{j}$. The cylinder represents the excavated volume, where $d$ is its depth and $R_{i}$ is the excavation radius (corresponding to the rim radius) in direction $i$.

the next section. An obvious candidate for the source of random variation which may produce this type of scaling is the strength heterogeneities in target materials (Watters, 2009). There may be multiple ways to motivate the hypothesis that the radial distance of crater growth is proportional to sums of a randomly-distributed quantity. We consider one of these in the following discussion, which assumes a simplified geometry.

We start by developing a scaling argument that considers the influence of strength heterogeneities on the shock wave and excavation flow. The model configuration is illustrated in Figure 8 , in which the volume excavated from a layer spanned by strength defects is represented by a cylinder of average radius $\bar{R}$ and of constant thickness $d$. We will focus on the energy required to excavate a fixed-width slice of material (shaded) of length $R_{i}$. In reality, crater cavities are bowl-shaped and the excavation occurs along "stream tubes" as described by the Maxwell Zmodel (Maxwell, 1977). We have simplified the geometry, although a similar argument can be constructed for the more complex geometry of stream tubes. We also do not explicitly address the decay of shock wave energy inside the radius $\bar{R}$.

We begin by assuming that the energy available to excavate the cylinder is initially directed equally in all directions. That is, for each slice $i$ there is an amount of energy $E_{i}$ available to compress, heat, fracture, and excavate material in the slice. It follows from energy conservation that this energy is equal to the sum of (a) the work done against gravity to lift the slice $\left(E_{G}\right)$, and (b) the mechanical energy $(W)$ expended in the deformation of material in the slice. The basic premise of the model can be summarized as follows. First, we assume that $E_{G}$ is proportional to the total mass and hence the total length of the slice. Second, the total mechanical energy, $W$, is proportional to a radial sum which depends on the mechanical properties of individual strength heterogeneities in the slice. If the target materials in a given direction are relatively weak, so that $W$ is relatively small, then a larger slice can be removed because there is more energy available. This implies that the final rim has a larger radius in the direction corresponding to slice $i$. Likewise, stronger materials in a given direction will result in a smaller radius.

We start developing the model more formally with a statement of energy conservation:

$$
0=E_{i}-E_{G}-W \longrightarrow E_{G}=E_{i}-W
$$

As mentioned, the term $E_{G}$ is proportional to the total mass of the slice. For a linear density $\mu$ of the slice (e.g., in units of $\mathrm{kg} / \mathrm{m}$ ), this is proportional to $\mu R_{i} g d$ : i.e., $E_{G}=\gamma \mu R_{i} g d$, where $R_{i}$ is the length of slice $i$, and $\gamma$ is a constant of proportionality. Substituting into equation (5) for $E_{G}$ and 
solving for $R_{i}$, we find:

$$
R_{i}=\frac{E_{i}-W}{\gamma \mu g d}
$$

The term $W$ is a sum of the energies, $\sum_{j=1}^{N} w_{j}$, required to compress, with associated damage and heating, the set of discrete volumes that make up the slice shown in Figure 8. As a result, the right-hand side of equation (6) can be expressed as:

$$
R_{i}=\frac{1}{\gamma \mu g d}\left(E_{i}-W\right)=\frac{1}{\gamma \mu g d} \sum_{j=1}^{N}\left(E_{i}-w_{j}\right)=\sum_{j=1}^{N} \lambda_{j},
$$

where $\lambda_{j} \equiv\left(E_{i}-w_{j}\right) / \gamma \mu g d$. That is, the right-hand side can be expressed as a sum of the random numbers $\lambda_{j}$, which have units of length and are drawn from an unspecified distribution. For example, if the values $w_{j}$ depend largely on the properties of preexisting target fractures, then the distribution of the $w_{j}$ (and hence $\lambda_{j}$ ) may reflect the distribution of fracture lengths, spacings, and orientations. (For example, recall that less energy is required to displace a block that is bounded by fractures aligned with the direction of excavation, as in Poelchau et al. (2009) and Watters et al. (2011).) In what follows, we will consider both a uniform and normal distribution for the $\lambda_{j}$.

For the cylinder as a whole (for all slices), if the $\lambda_{j}$ are normally distributed, the standard deviation $\sigma_{R}$ of the $R_{i}$ is given by: $\sigma_{R} \sim \sqrt{N \delta \lambda^{2}}$ where $\delta \lambda$ is the standard deviation of the distribution of the values $\lambda_{j}$ of which $\bar{\lambda}$ is the average. Substituting $N \approx R_{i} / \bar{\lambda} \approx \bar{R} / \bar{\lambda}$, we find:

$$
\sigma_{R} \sim \delta \lambda \sqrt{\frac{\bar{R}}{\bar{\lambda}}}=\beta \bar{R}^{\frac{1}{2}}
$$

where $\beta \equiv \delta \lambda / \sqrt{\bar{\lambda}}$ is a constant. By contrast, if the $\lambda_{j}$ are uniformly distributed, then $\delta \lambda$ represents the range of the distribution of the values $\lambda_{j}$, rather than the standard deviation. We use the same symbol for both, where it is understood to represent the standard deviation in the case of a normal distribution, and the range in the case of a uniform distribution. In both cases, the radial deviation will scale as $\bar{R}^{\frac{1}{2}}$. Since $D=2 \bar{R}$, this implies $\sigma_{R} \sim D^{\frac{1}{2}}$, consistent with the limiting case of well-preserved simple craters in strong targets (Section 4.1).

\subsection{Monte Carlo radial sum model: fixed defect size}

The hypothesis that $R_{i}$ is proportional to a sum of random numbers, which is motivated by the observed scaling among well-preserved simple craters in strong targets, can be used to construct artificial planforms using a simple Monte Carlo approach. This is achieved by tracing the end points of rays whose lengths are computed from the sum of numbers sampled from a normal or uniform distribution. An example of this approach is shown in Figure 7C, where the planform in question would result from connecting the endpoints of rays. Such planforms would not be realistic, however, since real crater planforms are typically close to convex, as shown in the previous section (see Figure 5). A more realistic outline is obtained by computing the convex hull of the radial-sum outlines: the smallest convex polygon that encompasses the ray end points. This model is called the "Convex Hull of a Radial Sum" model, or "CHORS" for short. This is illustrated by the solid black outlines of the planforms in part $\mathrm{C}$ of Figure 7.

The convex hull step is required for two reasons. First, the overall paucity of craters with highly concave planforms indicates one of two things: (1) that the expanding crater planform 
is approximately convex in all stages of growth, or (2) that concavities in the expanding crater planform - zones that are "left behind" by the excavation flow in directions where excavation is the least efficient - are intrinsically unstable and slump in the early modification stage. Evidence for this process has been documented in Endurance Crater at Meridiani Planum on Mars and at Barringer Crater on the Earth (Watters et al., 2011). Second, the excavation flow along one model "ray" is not independent of the flow along its immediate neighbors because the rays cross similar volumes in the target. That is, we take the convex hull because the final radius in any direction is to some extent correlated with that of its neighbors on account of (1) partially overlapping slices whose mechanical properties are not completely independent, and (2) the early-time modification (slumping) that affects some fraction of the crater wall. The convex hull step in our model is a simple representation of these intrinsic correlations that produce a preponderance of convex crater planforms.

These correlations imply that the size scaling derived in the foregoing argument may be different from $\sigma_{R} \sim D^{\frac{1}{2}}$, since that argument implicitly assumed no correlations between the sums that determine the $R_{i}$. One of the model's free parameters is $\Delta \phi$, the angular separation between model "rays" along which the radial sums are computed. In model calculations, provided this is a constant between between $\Delta \phi=\pi / 256$ and $\Delta \phi=\pi / 6$, we find that the power-law exponent is $m=0.63 \pm 0.3$. That is, for values of $\bar{R}$ that are small multiples of $\bar{\lambda}$, the radial deviation is reduced more significantly by taking the convex hull, thereby increasing $m$. For larger values of $\Delta \phi$ (i.e., fewer summation rays) we find $m>0.6$. By contrast, in the limit as $\Delta \phi \rightarrow 0$, we find $m \rightarrow 0.5$, consistent with the scaling of a sum of random numbers (Section 5.3). We also find this result if $\Delta \phi$ is allowed to increase linearly with the average radius $\bar{R}$. That is, for small $\Delta \phi$ at large $\bar{R} / \bar{\lambda}$, the convex hull screens proportionally fewer rays and the scaling of radial deviation converges on $m=1 / 2$. The overall radial deviation, however, is always reduced after the convex hull step.

For a prescribed distribution of crater diameters (and hence of crater radii), the model's free parameters are the mean and width of the distribution from which the random numbers $\lambda_{j}$ are sampled (i.e., $\bar{\lambda}$ and $\delta \lambda$, respectively), as well as the aforesaid angular spacing $\Delta \phi$ between the summation rays. We have examined the case where the numbers $\lambda_{j}$ are sampled from a uniform and normal distribution, finding no significant difference in the size scaling, provided the standard deviations are equal. We also note that the value of $\bar{\lambda}$ can be considered a fundamental length scale in terms of which all other lengths can be expressed. For example, the crater radius can be expressed in dimensionless form as $R^{*} \equiv \bar{R} / \bar{\lambda}$, where $\bar{R}$ is the average planform radius (the average of the $R_{i}$ ). In this dimensionless form, the model has just two parameters: i.e., $\Delta \phi$ and the ratio of the distribution width to $\bar{\lambda}$, which we call $\delta \lambda^{*} \equiv \delta \lambda / \bar{\lambda}$.

We find that the power-law scaling of radial deviation (the exponent $m$ in $\sigma_{R}=a D^{m}$ ) is unaffected by the value of $\delta \lambda^{*}$, although the overall magnitude, dictated by the coefficient $a$, is of course significantly affected (i.e., $a$ increases with increasing $\delta \lambda^{*}$ ). Increasing $\bar{\lambda}$ also increases $a$ but does not affect $m$, since $m$ is an intrinsic property of radial sum models. In the model comparisons described in the next section, we set $\delta \lambda^{*}=1$ and allow $\bar{\lambda}$ to vary when fitting the model to the measured distributions. We emphasize again that this choice does not affect the power-law scaling of $\sigma_{R}$ with diameter. That is, the only parameter which slightly influences $m$ is the scaling or absolute size of $\Delta \phi$. By contrast, there are two parameters which determine $a$ : these are the width and mean of the distribution of the $\lambda_{j}$, or as we have we have chosen to express them, the mean $(\bar{\lambda})$ and the ratio of width/mean $\left(\delta \lambda^{*} \equiv \delta \lambda / \bar{\lambda}\right)$. 
Table 4: CHORS model parameters $\Delta \phi$ and $\bar{\lambda}$ that minimize the Anderson-Darling statistic for the comparison with $\sigma_{R}$ measured from HiRISE-simple rim planforms (MM, $D \geq 100 \mathrm{~m}$; see row 8 of Table 2: $m=0.649, a=0.161$ ). For each statistical realization, 1000 planform craters were generated. Varying $\Delta \phi=\pi / 256$ to $\Delta \phi=\pi / 8$ we find that $\bar{\lambda}$ varies from $18 \mathrm{~m}$ to $6 \mathrm{~m}$. The exponent $m$ of the model radial deviation ranges from $m \approx 0.59$ to $m \approx 0.67$ for uniformly-distributed defect lengths, and 0.60 to 0.63 for normally-distributed defect lengths, while the coefficient $(a)$ varies from 0.122 to 0.226 .

\begin{tabular}{lllll}
$\Delta \phi$ & distribution & $\bar{\lambda}(\mathrm{m})$ & $m$ & $a$ \\
\hline$\pi / 256$ & uniform & 18 & 0.662 & 0.122 \\
$\pi / 256$ & normal & 18 & 0.631 & 0.153 \\
$\pi / 128$ & uniform & 15 & 0.630 & 0.157 \\
$\pi / 128$ & normal & 14 & 0.621 & 0.164 \\
$\pi / 64$ & uniform & 14 & 0.625 & 0.175 \\
$\pi / 64$ & normal & 11 & 0.619 & 0.169 \\
$\pi / 32$ & uniform & 9 & 0.603 & 0.199 \\
$\pi / 32$ & normal & 10 & 0.613 & 0.193 \\
$\pi / 16$ & uniform & 7 & 0.621 & 0.183 \\
$\pi / 16$ & normal & 8 & 0.611 & 0.206 \\
$\pi / 8$ & uniform & 6 & 0.593 & 0.240 \\
$\pi / 8$ & normal & 6 & 0.602 & 0.226 \\
\hline
\end{tabular}

\section{Comparison of synthetic and measured planforms}

Owing to the measured dependence of radial deviation on crater size, we must be very careful when comparing modeled and measured planforms to ensure that the size distributions are identical. We have therefore performed the following steps: (i) we computed the cumulative distribution function (CDF) of the diameters of the measured population; (ii) we computed a linear interpolation of the CDF so that it is uniformly sampled; and then (iii) we have fit a high-order polynomial to the result. Then, (iv) this polynomial was differentiated to recover a probability density function (PDF) from which the model crater diameters were chosen using von Neumann rejection sampling. Note that step (ii) was required so that widely-spaced points were fit with a smooth curve in step (iii). In what follows, we compare model results in HiRISE-simple as well as HRSC-complex and LROC-complex.

\subsection{Simple craters}

As mentioned in Section 1, there is significant evidence that the planforms of many simple craters are primarily determined by asymmetries in the excavation flow (Shoemaker, 1960; Roddy, 1978; Poelchau et al., 2009; Watters et al., 2011). We therefore compared the results for HiRISE-simple (MM subset, for $D \geq 100 \mathrm{~m}$ ) to the CHORS model. Our approach was to find appropriate model parameters by minimizing the difference between the modeled and measured distributions of radial deviation. We considered multiple values of the angular spacing between rays: $\Delta \phi=\pi / 2^{p}$ for $p=2$ to 9 . After setting $\delta \lambda^{*}=1$, for each value of $\Delta \phi$ we computed the CDFs of the modeled and measured values of $\sigma_{R}$ and $a / b$, finding the length scale $\bar{\lambda}$ of model strength defects that minimized the Anderson-Darling statistic. The results are reported in Table 4 using a sample size of 1,000 synthetic planforms. 

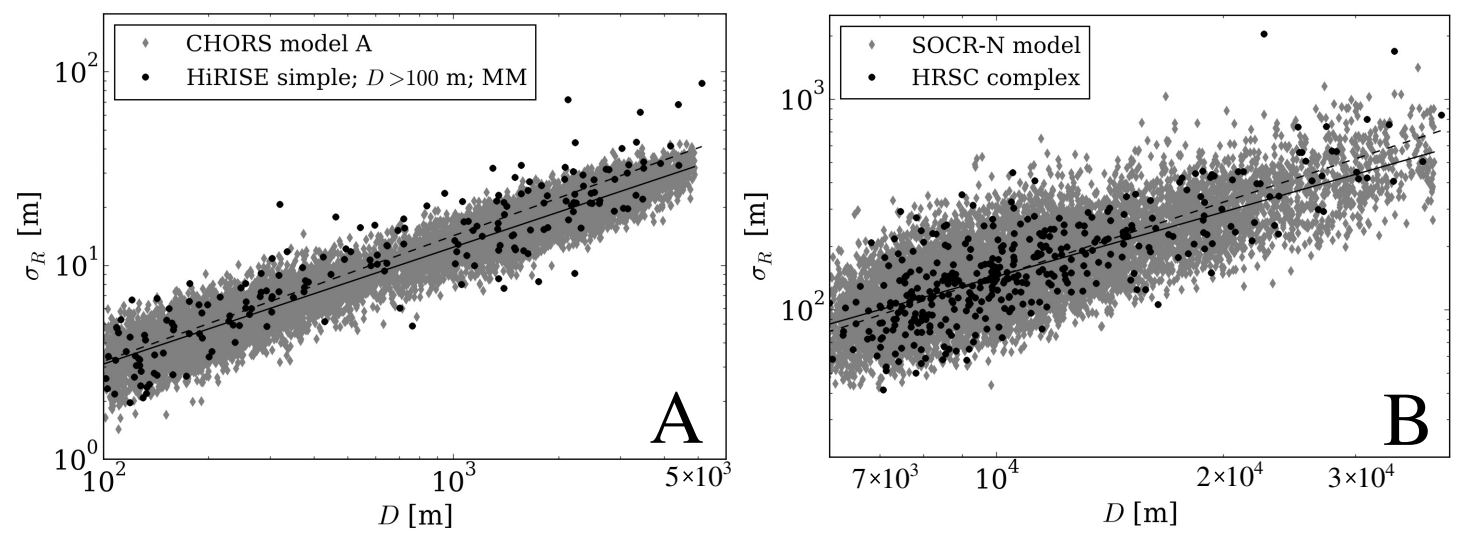

Figure 9: Radial deviation $\left(\sigma_{R}\right)$ versus diameter $(D)$ for measured planforms (black circles) and synthetic planforms (gray diamonds). (A) Comparison of well-preserved martian simple craters (HiRISE-simple, at most "moderately modified", $N=285)$ and planforms generated using the CHORS model A $(N=10,000)$. (B) Comparison of well-preserved martian complex craters (HRSC-complex, $N=382$ ) and planforms generated using the SOCR-N model (with normallydistributed segment lengths, $N=10,000$ ). In both cases, the dashed line was fit to measured rim planforms and the solid line to model planforms. See Tables 2 and 4 for the values of fit parameters.

Figures 9A through 11A show results from two versions of the CHORS model, each with $\Delta \phi=\pi / 8$. We chose a large value of $\Delta \phi$ because this implies a small number of rays, in which case a large number of model realizations can be generated quickly. A larger number of rays implies a larger $\bar{\lambda}$, while the distribution of morphometric quantities remains largely unchanged and the exponent of the size-scaling of $\sigma_{R}$ is only moderately affected (Table 4). Using 10,000 synthetic planforms, we have refined the value of $\bar{\lambda}$ still further. For "CHORS model A," we find $\bar{\lambda}=4 \mathrm{~m}$ by minimizing the Anderson-Darling statistic in the comparison between measured and model distributions of $\sigma_{R}$. For "CHORS model B" we find $\bar{\lambda}=8 \mathrm{~m}$ by minimizing the Anderson-Darling statistic in the comparison between measured and modeled distributions of $a / b$. We have accomplished these fits using the quantities measured from rim planforms in HiRISE-simple (craters in MM with $D \geq 100 \mathrm{~m}$ ) rather than cavity planforms. Because the overall radial deviation of rim planforms is larger than for cavity planforms, we do not expect a good match to both distributions at once.

As mentioned earlier, for any fixed value of $\Delta \phi$ within a large range $(\pi / 256<\Delta \phi<\pi / 6)$, we find that model planforms follow $\sigma_{R} \sim D^{m}$ for $m \approx 0.63 \pm 0.3$, consistent with the measured sizedependent scaling for simple craters with $D \geq 100 \mathrm{~m}$ that exhibit good preservation (Tables 2 and 4). Measurements of $\sigma_{R}$ computed from synthetic planforms from CHORS model A are plotted in Figure 9A, which shows that the scatter in $\sigma_{R}$ is similar between model and observations. Some of the measured values of $\sigma_{R}$ plot above as well as below the model range. Planforms plotting significantly above the model range (high asymmetry) may be the result of highly oblique impacts or cratering in highly anisotropic materials. Likewise, planforms plotting significantly below the model range (high symmetry) may have formed in isotropic or very low-strength targets and are relatively unaffected by strength defects that largely account for deviations from symmetry in the main population. It is important to bear these two potential sources for outliers in mind while making the comparison. 

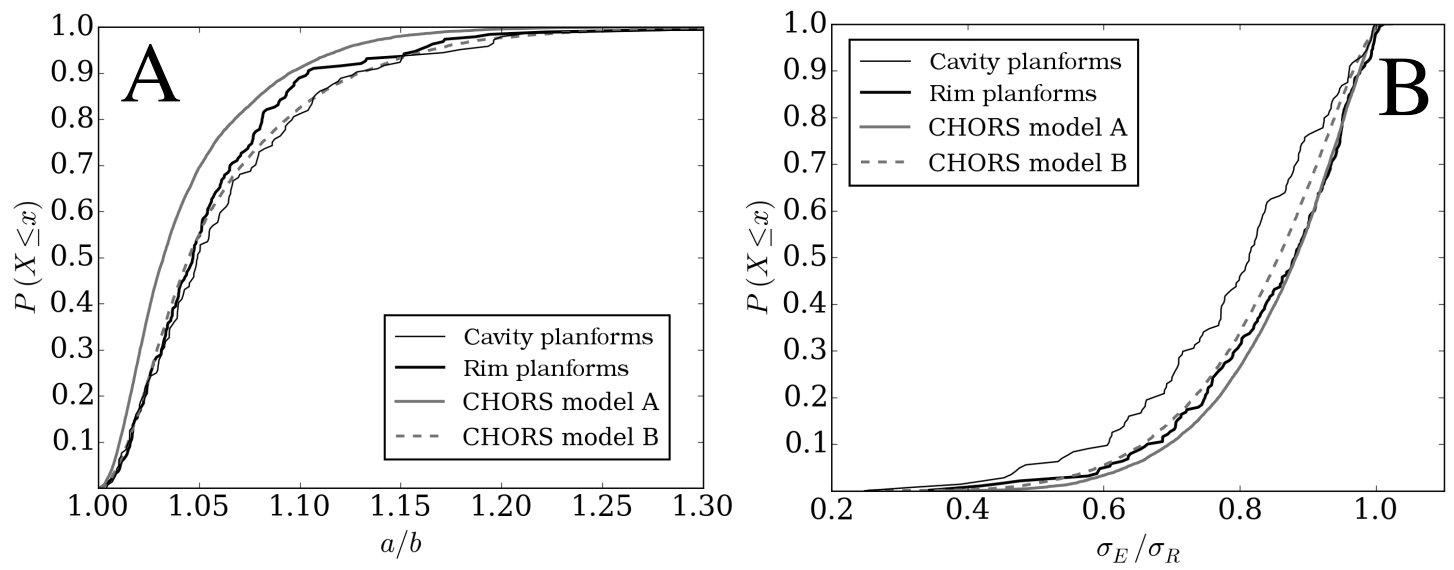

Figure 10: Cumulative distribution functions (CDF) for two measures of planform asymmetry, computed for measured and synthetic planforms. (A) Measured planform ellipticity $(a / b)$ in HiRISE-simple $(D \geq 100 \mathrm{~m}$, at most "moderately modified" (MM), $N=284)$ and synthetic planforms in CHORS model A $\left(\delta \lambda^{*}=1, \Delta \phi=\pi / 8, \bar{\lambda}=4 \mathrm{~m}, N=10,000\right)$ and CHORS model B (identical except $\bar{\lambda}=8 \mathrm{~m}$ ). (B) Residual fraction $\left(\sigma_{E} / \sigma_{R}\right)$ for the same measured and synthetic crater planforms. See text for discussion.

Figure 10A shows the CDF for two other measures of asymmetry discussed in Section 3.3: the planform ellipticity $(a / b)$, and the residual deviation $\left(\sigma_{E}\right.$, the root mean square radial deviation from the fitted ellipse). Recall that $\bar{\lambda}$ was selected so that CHORS model B fits the measured CDF for $a / b$ as closely as possible, while CHORS model A is the closest fit to the CDF for the overall radial deviation, $\sigma_{R}$. Part $\mathrm{B}$ of this figure shows the comparison for the CDF of residual deviation normalized by overall radial deviation.

Figure 10 helps to illustrate how the different sources of asymmetry complicate the comparison of synthetic and measured planforms. It is clear from part A that a major part of the asymmetry derives from an overall elongation, which will be significantly affected by the small fraction of planforms resulting from highly oblique impacts. Part B of the figure shows that if we ignore this elongation by examining just the residual deviation, both models provide a good match to the CDF for rim planforms. Cavity planforms exhibit far less radial deviation overall, and so the model cannot be used to fit both simultaneously.

A clearer sense of the contribution of overall elongation to planform asymmetry can be appreciated from the distribution of dominant harmonics $\left(n_{0}\right)$. These are plotted for CHORS-A, CHORS-B, and HiRISE-simple in Figure 11A. This is a frequency histogram of dominant harmonics. For example, the fraction of rim planforms that are dominantly square-shaped is plotted for $n_{0}=4$. This plot reveals a significant difference between synthetic and measured planforms for the lowest-order harmonics, and especially for $n_{0}=2$, indicating an overall elongation. Also, CHORS model B planforms are slightly more often elongated (dominant harmonic $n_{0}=2$ ) when compared with model $\mathrm{A}$, consistent with the smaller values of $a / b$ for model $\mathrm{A}$, on average, in Figure 10A.

The fraction of $n_{0}=2$ planforms which occur in nature (in HiRISE-simple) exceeds both model results by $12 \%$ to $15 \%$. The CHORS models, which do not incorporate the effects of oblique impact, suggest that asymmetries arising from the radial sum variations account for most 

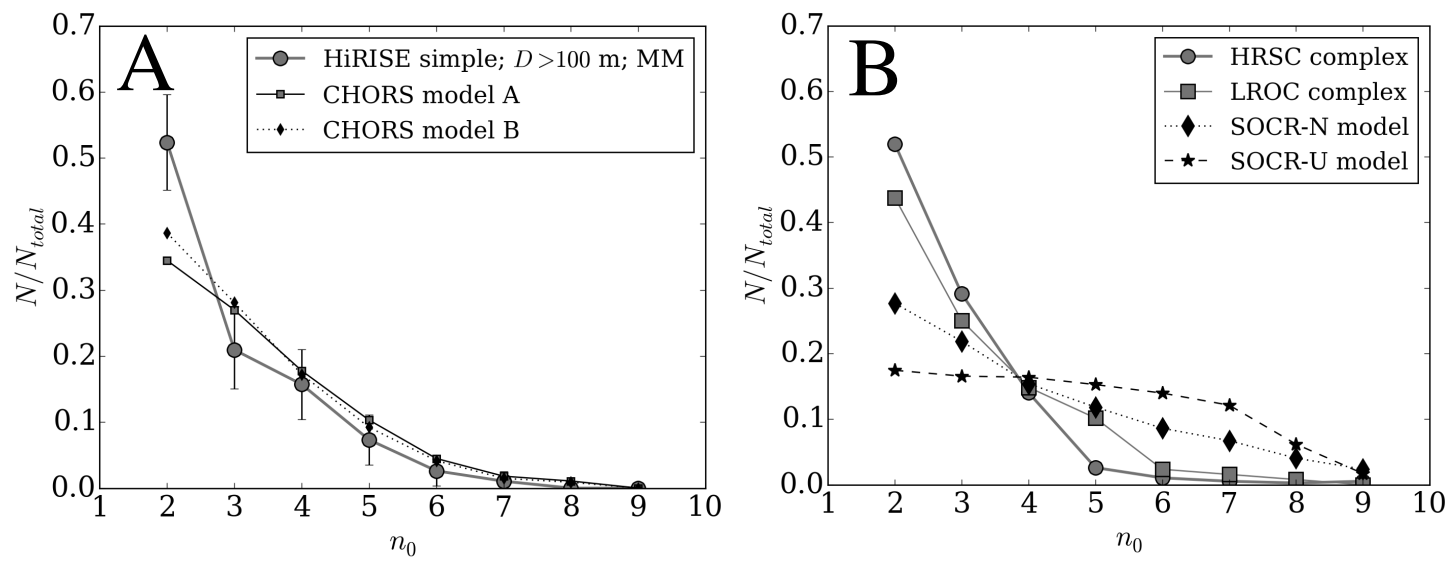

Figure 11: Distribution of dominant harmonics (or "shape distribution") of measured and synthetic crater planforms. Each plot shows the fraction of crater planforms whose dominant harmonic is 2 (elongated), 3 (triangular), 4 (squareshaped), and so on. (A) Comparison of HiRISE-simple rim planforms ("moderately modified", $D \geq 100 \mathrm{~m}$ ) and CHORS models A and B. The largest discrepancy occurs in the second harmonic, as expected, owing to a small population that is elongated by oblique impact. The uncertainties plotted for HiRISE-simple represent the $2 \sigma$ variation for each harmonic in a bootstrap analysis with 10,000 realizations. (B) Comparison of HRSC-complex and LROC-complex with the SOCR model for the case of normally-distributed segment lengths (SOCR-N) and uniformly-distributed segment lengths (SOCR-U). Uncertainties are not plotted to avoid overlapping error bars. For HRSC-complex, the largest uncertainty (from an identical bootstrap analysis) is \pm 0.05 for $n_{0}=2$; for LROC-complex, the largest uncertainty is \pm 0.09 , also for $n_{0}=2$.

but not all planforms with a dominant second harmonic. This may suggest that between $12 \%$ and $15 \%$ of planforms are influenced by the effects of oblique impact. Since the probability of an impact angle $\leq \theta$ is equal to $\sin ^{2} \theta$, this fraction of the population corresponds to impact angles smaller than $20^{\circ}$ to $23^{\circ}$ (Shoemaker, 1962). This is significantly larger than the $12^{\circ}$ estimated by Bottke et al. (2000). It is important to bear in mind, however, that Bottke et al. (2000) was only counting craters with $a / b \geq 1.1$. By contrast, craters having $n_{0}=2$ only means that the 2 nd harmonic is strongest, and may have ellipticity $a / b \leq 1.1$.

In summary, our results suggest that $n_{0}=2$ occurs significantly more often than one expects "by chance"; this excess may be largely the result of oblique impact. It is also worth recalling that laboratory experiments in Anderson et al. (2004b) found pronounced asymmetries in the excavation flow for impact angles as large as $30^{\circ}$ from horizontal. On the other hand, Figure 10A shows that roughly $10 \%$ of crater rim planforms in HiRISE-simple exhibit $a / b>1.1$. This is also true of about $11 \%$ of craters in LROC-complex, and roughly $6.5 \%$ of craters in HRSC-complex. We cannot completely rule out that our relatively small sample populations simply happen to comprise more elongated craters than other, larger databases.

\subsection{Complex craters}

As mentioned in Section 1, there is significant evidence that complex crater planforms are primarily influenced by a process of crater collapse (Melosh and Ivanov, 1999). We therefore compare the results for HRSC-complex and LROC-complex to the SOCR model, in which planforms are constructed by connecting line segments whose lengths are sampled from an assumed 
distribution. As discussed in the previous section, this comparison is motivated by the idea that the length scale of deviations from circular symmetry (relatively straight wall segments) may reflect the size distribution of strength defects influencing the local collapse of crater walls. Moreover, this is accomplished by modifying a symmetric shape (by segmenting or "beveling" a circle) and in this sense may bear a closer resemblance to the collapse process that forms the planforms of complex craters. The SOCR model is prescribed by the characteristics of the distribution from which segment lengths $\ell_{j}$ are chosen. For the "SOCR-N" model, these segment lengths are selected from a normal distribution, and a uniform distribution for "SOCR-U."

The only free parameter of the SOCR models is the scaling of the mean size of segment lengths: $\bar{\ell}=k \bar{R}$ for mean radius $\bar{R}$. We estimated $k$ by minimizing the Anderson-Darling statistic, computed for the comparison between synthetic and measured planforms. We chose HRSCcomplex for this analysis, because it has the larger number of planforms, finding $k=0.47 \pm 0.01$ for both SOCR-N and SOCR-U.

Figure 9B is a plot of the radial deviation as a function of diameter for the craters in HRSCcomplex and SOCR-N. This illustrates that the relatively large breadth of the scatter at small diameters $\left(D<10^{4.1} \approx 12.6 \mathrm{~km}\right)$ is also apparent in the model results, and is therefore likely to be a consequence of the relatively high abundance of craters with small diameters in our data set (i.e., there are more well-preserved complex craters at small sizes).

It is important to emphasize that, unlike the CHORS model, in which the size-dependent scaling is an intrinsic property, we have prescribed the scaling for SOCR by setting $\bar{\ell}=k R^{m}$

for $m=1$. Our discussion therefore focuses on the comparison between the distributions of dominant harmonics for synthetic and measured planforms, as shown in Figure 11B.

Like HiRISE-simple, the distribution of dominant harmonics for LROC-complex and HRSCcomplex indicate that over $40 \%$ of planforms are dominated by the second harmonic, unlike any of the model results. Once again, we suggest this is likely a consequence of highly oblique impacts, which increase the number of craters with an overall elongation. Also in all three distributions, the fraction that are square-shaped (dominant 4 th harmonic) is approximately $15 \%$. The sixth and higher harmonics are all less than 5\% of the overall distribution. The largest disagreements occur in the odd-numbered harmonics 3 and 5 (with only one symmetry plane), where HiRISE-simple has the most triangles and LROC-complex has the most pentagons. It is worth emphasizing that the planform shape distributions are very similar in the even-numbered harmonics for simple and complex craters on Mars. This is despite clearly different size-scaling of $\sigma_{R}$ and the very different processes thought to be responsible for determining the final shape of craters in each population.

The SOCR-N and SOCR-U models are a poor match to the measured distribution of dominant harmonics for complex craters. All three data sets and all models agree that the fraction of squareshaped craters is $\approx 15 \%$. By contrast, the disagreement is significant for all other harmonics, with both SOCR-U and SOCR-N anticipating many more craters with higher dominant harmonics and fewer with lower harmonics. We suggest this is a consequence of selecting segment lengths from a symmetric distribution, leading to a large abundance of shapes which exhibit multiple lines of symmetry and with more power concentrated in high-order harmonics. We find that the agreement for CDFs of other measured quantities, such as $\sigma_{E}$ and $\Omega$, is also poor (not shown).

\section{Discussion}

The Monte Carlo radial sum model can be used to accurately construct the distribution of simple crater planforms observed in nature, with the exception of some outliers, which may 
have formed in completely strengthless targets or oblique impacts. The distribution of dominant harmonics (Figure 11) illustrates the similarity between the distributions for HiRISE-simple, LROC-complex, and HRSC-complex. That is, while the overall distribution of shapes is similar, the diameter scaling of deviations from symmetry is different. Other features of the shape distribution for complex and simple craters are also very similar. The CDFs for $\sigma_{E} / \sigma_{R}$ and $a / b$ are virtually identical (not shown), as well as $\sigma_{C} / \sigma_{R}$ (when comparing to cavity planforms in HiRISE-simple; see Figure 5B).

The foregoing observations suggests that, with modifications, the CHORS model may also serve as a good representation of the complex crater distribution, were it not for the $\sigma_{R}$ vs. $D$ scaling. A promising candidate for such a model begins with rays of identical length (circular symmetry) and then adds a length to each ray, chosen from a normal distribution whose mean value is a constant fraction of the crater diameter (to guarantee the correct scaling). That a normal distribution results from the radial sum in CHORS is a consequence of the Central Limit Theorem. Preliminary experiments with this modified "randomized radial retreat" model indicates very good agreement with the distribution of complex crater shapes. We leave the further development of this model for future work.

This leaves open the question of why the characteristic length of deviations from symmetry scales linearly with diameter for complex craters. We suggest this may be a simple consequence of the scaling of the largest fractures, slumps, and faults with respect to the overall size of a negative load and related stress field. If correct, then we might expect the characteristic length of variations in many kinds of collapse features to scale linearly with the overall size of related depressions, such as in calderas and sink holes. To the extent that terrace widths are characteristic of the radial length scale of collapse structures in complex craters, the scaling of terrace width with crater diameter may hold a clue. Previous authors have found that the widths, $W$, of firstorder terraces scale with diameter of lunar complex craters as $W=0.09 D^{0.87}$ (for quantities in km; Pearce and Melosh (1986)). By inspection of Figure 2 in Pearce and Melosh (1986) the dependence is closer to linear for $D<100 \mathrm{~km}$.

As mentioned, previous work has shown that preexisting defects tend in some cases to align with crater walls (Öhman et al., 2008; Kumar and Kring, 2008; Watters, 2009; Beddingfield et al., 2016), as clearly shown in the case of small modified craters in Figure 1E-F. As noted earlier, the scaling exponent $m$ tends to increase among the more significantly modified craters in HiRISE-simple. That is, as modification proceeds, the scaling of deviations from symmetry approaches that of complex craters, whose planforms are widely believed to result from a collapse process. For this reason, it is extremely important to pay close attention to preservation state and, if possible, impact crater structure, when drawing conclusions about whether planforms are primarily influenced by an excavation flow that exploits strength defects - such as anisotropies in shear strength (Poelchau et al., 2009; Watters et al., 2011) or thrusting along preexisting fractures (Öhman et al., 2008) or overturn along preexisting fractures (Kumar and Kring, 2008)) - versus the processes that control short- and long-term modification.

For at least two impact craters, it has been shown through structural analysis that the transient cavity had a concave planform (Barringer and Endurance craters, Shoemaker (1960) and Watters et al. (2011)), demonstrating that the excavation proceeded farther in some directions than others, effectively "leaving behind" some portions of the crater walls during crater growth. This argues strongly for an excavation flow that dominates the formation of asymmetries at early times. On the other hand, recent work by our group has shown that rim collapse is more pronounced for craters exceeding a threshold diameter $(D \approx 1 \mathrm{~km}$ on Mars, Watters et al. (2015)), suggesting that early modification may to some extent mask the signature of asymmetric excavation. In the 
present work, we do not detect strong signs of a transition in planform morphometry at $D \approx 1$ $\mathrm{km}$ on Mars.

The present work might be extended in a number of fruitful directions. Possibly the most pressing task is to conduct a similar analysis of simple craters on the Moon, to determine whether a similar scaling is observed despite the generally thicker regolith. For example, our work predicts different scaling for the stronger maria (smaller $m$ ) when compared with weaker highlands materials. Similar analyses might be conducted for simple and complex craters on low-gravity worlds like Vesta and ice-dominated Ceres, to identify the influence of weaker, volatile-rich targets upon the distribution and scaling. Finally, it could be worthwhile to consider the use of fully three-dimensional measures of the departure from radial symmetry, such as been proposed by several studies in the last two decades (Wallis and McBride, 2002; Wallis et al., 2002, 2005; Tabares-Rodenas et al., 2013). In future, fully 3-D hydrocode models, incorporating strength heterogeneities with realistic variation, might be used to understand the size scaling; some work in this direction has already begun (Plesko, 2015). A more realistic implementation of discrete element modeling in Watters et al. (2011) might also be attempted.

It may also be interesting to examine in detail how the maximum scale or distribution of size and spacing of fractures in target materials influences the departure from symmetry of wellpreserved simple craters in a specific diameter range. Unfortunately, we anticipate it will be challenging to find locations on Mars where fractures are clearly visible and where there is sufficient HiRISE coverage to make a statistically significant number of measurements to determine this relationship. At present, we suggest that Meridiani Planum is the most ideal setting in which to attempt this investigation. In a similar vein, by using morphometry from high-resolution imagery of a region with well-characterized layering of rocks with prominent strength variations, it may be possible to estimate the depth of target materials that significantly influence the planform, as suggested by Fulmer and Roberts (1963).

\section{Conclusions}

We have presented morphometric analyses of well-preserved simple and complex impact craters on Mars and the Moon. We have also described Monte Carlo models of synthetic crater planform populations based on simple physical and geometrical principles. This work indicates the following conclusions:

1. The radial deviation ( $\sigma_{R}$, standard deviation of radius) of the rim and cavity planforms of well-preserved simple craters on Mars follows a power law with exponent $m \approx 0.65$ (for $D \geq 100 \mathrm{~m}$ ). This exponent is smaller for the best-preserved craters, as well as craters forming in strong targets $(m=0.5-0.6)$.

2. As a fraction of average crater radius, the radial deviation $\left(\sigma_{R} / \bar{R}\right)$ reaches a maximum at or below $D \approx 100 \mathrm{~m}$. For smaller diameters, $\sigma_{R} / \bar{R}$ decreases or remains constant. This may reflect the fact that, on average, many small craters form entirely in low-strength regolith or strengthless sand, and hence exhibit greater symmetry.

3. By contrast with well-preserved simple craters, the diameter dependence of $\sigma_{R}$ for complex craters indicates $m \approx 1$ for the Moon and Mars (ranging from 0.9 to 1.2), consistent with the results of earlier studies using similar metrics (of radial deviation rather than "circularity," which is problematic for reasons discussed in Section 1.3).

4. The observed size scaling rules out the hypothesis that deviations from symmetry are simply equal to the uniform and constant size of preexisting strength heterogeneities, since this assumption predicts $m=-1$. 
5. Some heavily-modified simple craters exhibit straight wall segments which are aligned with regional joints (see Figure 1E-F and Watters (2009)), as expected from the long-term collapse of rim walls along preexisting planes of weakness.

6. We developed a Monte Carlo model based on a physically-motivated scaling argument, which exhibits power-law scaling of $\sigma_{R}$ with diameter (exponent $m=0.50-0.66$ ). This model accurately reproduces many features of simple crater planforms observed in nature. When compared with the measured shape distribution, this model suggests that $12 \%-15 \%$ of primary impact craters may owe their elongation to the influence of oblique impact.

7. We find no universal tendency for radius to be correlated with rim height in well-preserved simple craters, as might be expected if there is a tendency for flap hinge uplift to be correlated with radius (Poelchau et al., 2009).

8. Simple craters forming in strong targets tend to exhibit a higher degree of concavity, as expected if concave transient cavity planforms form as a result of shear strength anisotropies (e.g., Watters et al. (2011)).

9. The distribution of even-numbered dominant harmonics for well-preserved simple and complex craters on Mars are similar, despite involving very different processes. In the three populations examined in this study, square-shaped craters comprise $15 \pm 2 \%$ of the population.

10. The planforms of small complex craters on Mars $(D<10 \mathrm{~km})$ and the Moon $(D<40 \mathrm{~km})$ are more strongly dominated by low-order harmonics when compared with large craters, consistent with previous results.

11. Concavities make up a far more significant fraction of the radial deviation for complex craters with relatively large diameters on Mars $(D>10 \mathrm{~km})$ and the Moon $(D>40 \mathrm{~km})$. On both worlds, complex craters exhibit a higher degree of concavity in weaker target materials.

\section{Acknowledgments}

We gratefully acknowledge grants from Wellesley College alumnae funds to support the work of L.G. (Schiff fellowship), R.G., and M.F. (Malone Summer Research Grant). We are grateful to Trent Hare (USGS) for GIS-related advice and for providing a raster version of the Tanaka et al. (2014) map. We also thank Bradford Hager, Brandon Johnson, Cassandra Pattanayak, Michael Poelchau, Jamie Riggs, and Maria Zuber for helpful discussions, from which this work has benefited. We are grateful to David H. Baker and one anonymous reviewer for very helpful reviews of our manuscript. Finally, all of the work presented in this paper was accomplished using free and open source software: we are grateful to the developers of the Ames Stereo Pipeline, GNU core utilities, Emacs, Inkscape, QGIS, Python, Scientific and Numerical Python, Matplotlib, LTEX, and Debian GNU/Linux. A database containing the quantities used to produce the tables and graphs in this paper is available for download as supplementary materials. Readers may also contact the corresponding author (wwatters@wellesley.edu) to request a copy of these data.

Aittola, M., Öhman, T., Leitner, J. J., Kostama, V.-P., Raitala, J., Feb. 2010. The structural control of venusian polygonal impact craters. Icarus 205, 356-363.

Aittola, M., Öhman, T., Leitner, J. J., Raitala, J., Oct. 2007. The Characteristics of Polygonal Impact Craters on Venus. Earth Moon and Planets 101, 41-53.

Anderson, J. A., Sides, S. C., Soltesz, D. L., Sucharski, T. L., Becker, K. J., Mar. 2004a. Modernization of the Integrated Software for Imagers and Spectrometers. In: Mackwell, S., Stansbery, E. (Eds.), Lunar and Planetary Institute Science Conference Abstracts. Vol. 35. p. 2039.

Anderson, J. L. B., Schultz, P. H., Heineck, J. T., Feb. 2004b. Experimental ejection angles for oblique impacts: Implications for the subsurface flow-field. Meteoritics and Planetary Science 39, 303-320.

Andrews-Hanna, J. C., Zuber, M. T., 2010. Elliptical craters and basins on the terrestrial planets. Geological Society of America Special Papers 465, 1-13. 
Baker, D. M. H., Head, J. W., Sep. 2013. New morphometric measurements of craters and basins on Mercury and the Moon from MESSENGER and LRO altimetry and image data: An observational framework for evaluating models of peak-ring basin formation. Planetary and Space Science 86, 91-116.

Baker, D. M. H., Head, J. W., Neumann, G. A., Smith, D. E., Zuber, M. T., Mar. 2012. The transition from complex craters to multi-ring basins on the Moon: Quantitative geometric properties from Lunar Reconnaissance Orbiter Lunar Orbiter Laser Altimeter (LOLA) data. Journal of Geophysical Research (Planets) 117, 0.

Barlow, N. G., Mar. 2004. Martian subsurface volatile concentrations as a function of time: Clues from layered ejecta craters. Geophysical Research Letters 31, 5703.

Barlow, N. G., 2015. Characteristics of impact craters in the northern hemisphere of Mars. Geological Society of America Special Papers 518, SPE518-03.

Beddingfield, C. B., Burr, D. M., Tran, L. T., 2016. Polygonal impact craters on Dione: Evidence for tectonic structures outside the wispy terrain. Icarus 274, 163-194.

Bottke, W. F., Love, S. G., Tytell, D., Glotch, T., May 2000. Interpreting the Elliptical Crater Populations on Mars, Venus, and the Moon. Icarus 145, 108-121.

Brandt, D., Reimold, W. U., 1995. The geology of the Pretoria Saltpan impact structure and the surrounding area. S. Afr. J. Geol. 98, 287-303.

Calef, F. J., Herrick, R. R., Sharpton, V. L., Oct. 2009. Geomorphic analysis of small rayed craters on Mars: Examining primary versus secondary impacts. Journal of Geophysical Research (Planets) 114 (E13), 10007.

Christensen, P., Jakosky, B., Kieffer, H., Malin, M., McSween, HarryY., J., Nealson, K., Mehall, G., Silverman, S., Ferry, S., Caplinger, M., Ravine, M., 2004. The thermal emission imaging system (THEMIS) for the Mars 2001 Odyssey mission. Space Science Reviews 110 (1-2), 85-130.

Collins, G. S., Elbeshausen, D., Davison, T. M., Robbins, S. J., Hynek, B. M., Oct. 2011. The size-frequency distribution of elliptical impact craters. Earth and Planetary Science Letters 310, 1-8.

Elbeshausen, D., Wünnemann, K., Collins, G. S., Nov. 2013. The transition from circular to elliptical impact craters. Journal of Geophysical Research (Planets) 118, 2295-2309.

Eppler, D. T., Ehrlich, R., Nummedal, D., Schultz, P. H., 1983. Sources of shape variation in lunar impact craters: Fourier shape analysis. Geological Society of America Bulletin 94, 274.

Eppler, D. T., Nummedal, D., Ehrlech, R., 1977. Fourier analysis of lunar crater shape - possible guide to impact history and lunar geology. In: Roddy, D. J., Pepin, R. O., Merrill, R. B. (Eds.), Impact and explosion cratering. Pergamon Press, New York, New York, pp. 511-526.

Fulmer, C. V., Roberts, W. A., 1963. Rock induration and crater shape. Icarus 2, 452-465.

Gault, D. E., Quaide, W. L., Oberbeck, V. R., 1968. Impact cratering mechanics and structures. In: French, B. M., Short, N. M. (Eds.), Shock Metamorphism of Natural Materials. Mono Book Corp, Baltimore, Maryland, pp. 87-99.

Gault, D. E., Wedekind, J. A., 1978. Experimental studies of oblique impact. In: Lunar and Planetary Science Conference Proceedings. Vol. 9 of Lunar and Planetary Science Conference Proceedings. pp. 3843-3875.

Geiger, L. M., May 2013. Statistical analysis of simple martian impact crater morphometry. Undergraduate honors thesis; http://repository.wellesley.edu/thesiscollection/136/, Wellesley College, 106 Central Street, Wellesley, MA 02481.

Gifford, A. W., Maxwell, T. A., 1979. Asymmetric terracing of lunar highland craters - Influence of pre-impact topography and structure. In: Hinners, N. W. (Ed.), Lunar and Planetary Science Conference Proceedings. Vol. 10 of Lunar and Planetary Science Conference Proceedings. pp. 2597-2607.

Gulick, S. P., Barton, P. J., Christeson, G. L., Morgan, J. V., McDonald, M., Mendoza-Cervantes, K., Pearson, Z. F., Surendra, A., Urrutia-Fucugauchi, J., Vermeesch, P. M., et al., 2008. Importance of pre-impact crustal structure for the asymmetry of the Chicxulub impact crater. Nature Geoscience 1 (2), 131-135.

Jaumann, R., Neukum, G., Behnke, T., Duxbury, T. C., Eichentopf, K., Flohrer, J., Gasselt, S. v., Giese, B., Gwinner, K., Hauber, E., Hoffmann, H., Hoffmeister, A., Köhler, U., Matz, K.-D., McCord, T. B., Mertens, V., Oberst, J., Pischel, R., Reiss, D., Ress, E., Roatsch, T., Saiger, P., Scholten, F., Schwarz, G., Stephan, K., Wählisch, M., the HRSC Co-Investigator Team, May 2007. The high-resolution stereo camera (HRSC) experiment on Mars Express: Instrument aspects and experiment conduct from interplanetary cruise through the nominal mission. Planet. Space Sci. 55, 928-952.

Kalynn, J., Johnson, C. L., Osinski, G. R., Barnouin, O., Jan. 2013. Topographic characterization of lunar complex craters. Geophysical Research Letters 40, 38-42.

Kring, D. A., Mar. 2015. Botanical Signature of Tectonic Fractures in the Target Rocks of Barringer Meteorite Crater, Arizona. In: Lunar and Planetary Science Conference. Vol. 46 of Lunar and Planetary Science Conference. p. 1036.

Kumar, P. S., Kring, D. A., 2008. Impact fracturing and structural modification of sedimentary rocks at Meteor Crater, Arizona. Journal of Geophysical Research: Planets (1991-2012) 113 (E9).

Losiak, A., Wilhelms, D. E., Byrne, C. J., Thaisen, K. G., Weider, S. Z., Kohout, T., O’Sullivan, K., Kring, D. A., 2009. A New Lunar Impact Crater Database. In: Lunar and Planetary Institute Science Conference Abstracts. Vol. 40. p. 1532.

Malin, M. C., Edgett, K. S., 2001. Mars global surveyor mars orbiter camera: interplanetary cruise through primary 
mission. Journal of Geophysical Research: Planets 106 (E10), 23429-23570.

Mandelbrot, B. B., 1983. The fractal geometry of nature. Vol. 173. Macmillan.

Maxwell, D. E., 1977. Simple Z model of cratering, ejection, and the overturned flap. In: Roddy, D. J., Pepin, R. O., Merrill, R. B. (Eds.), Impact and explosion cratering. Pergamon Press, New York, New York, pp. 983-1022.

McEwen, A. S., Banks, M. E., Baugh, N., Becker, K., Boyd, A., Bergstrom, J. W., Beyer, R. A., Bortolini, E., Bridges, N. T., Byrne, S., Castalia, B., Chuang, F. C., Crumpler, L. S., Daubar, I., Davatzes, A. K., Deardorff, D. G., DeJong, A., Alan Delamere, W., Dobrea, E. N., Dundas, C. M., Eliason, E. M., Espinoza, Y., Fennema, A., Fishbaugh, K. E., Forrester, T., Geissler, P. E., Grant, J. A., Griffes, J. L., Grotzinger, J. P., Gulick, V. C., Hansen, C. J., Herkenhoff, K. E., Heyd, R., Jaeger, W. L., Jones, D., Kanefsky, B., Keszthelyi, L., King, R., Kirk, R. L., Kolb, K. J., Lasco, J., Lefort, A., Leis, R., Lewis, K. W., Martinez-Alonso, S., Mattson, S., McArthur, G., Mellon, M. T., Metz, J. M., Milazzo, M. P., Milliken, R. E., Motazedian, T., Okubo, C. H., Ortiz, A., Philippoff, A. J., Plassmann, J., Polit, A., Russell, P. S., Schaller, C., Searls, M. L., Spriggs, T., Squyres, S. W., Tarr, S., Thomas, N., Thomson, B. J., Tornabene, L. L., Van Houten, C., Verba, C., Weitz, C. M., Wray, J. J., Jan. 2010. The High Resolution Imaging Science Experiment (HiRISE) during MRO’s Primary Science Phase (PSP). Icarus 205, 2-37.

Melosh, H., Ivanov, B., 1999. Impact crater collapse. Annual Review of Earth and Planetary Sciences 27 (1), 385-415.

Melosh, H. J., 1979. Acoustic fluidization: A new geologic process? Journal of Geophysical Research: Solid Earth 84 (B13), 7513-7520.

URL http://dx.doi.org/10.1029/JB084iB13p07513

Melosh, H. J., 1989. Impact cratering: A geologic process. Oxford University Press, 253 p.

Moratto, Z. M., Broxton, M. J., Beyer, R. A., Lundy, M., Husmann, K., Mar. 2010. Ames Stereo Pipeline, NASA's Open Source Automated Stereogrammetry Software. In: Lunar and Planetary Institute Science Conference Abstracts. Vol. 41. p. 2364

Murray, J. B., Guest, J. E., 1970. Circularity of craters and related structures on the Earth and Moon. Modern Geology $1,149-159$.

Oberbeck, V. R., Aoyagi, M., Murray, J. B., 1972. Circularity of Martian craters. Modern Geology 3, 195-199.

Öhman, T., Aittola, M., Korteniemi, J., Kostama, V.-P., Raitala, J., 2010. Polygonal impact craters in the solar system; observations and implications. Geological Society of America Special Papers 465, 51-65.

Öhman, T., Aittola, M., Kostama, V., Raitala, J., 2005. The preliminary analysis of polygonal impact craters within greater Hellas region, Mars. In: Koeberl, C., Henkel, H. (Eds.), Impact Tectonics. Springer, Berlin, pp. 131-143.

Öhman, T., Aittola, M., Kostama, V.-P., Hyvärinen, M., Raitala, J., Aug. 2006. Polygonal impact craters in the Argyre region, Mars: Evidence for influence of target structure on the final crater morphology. Meteoritics and Planetary Science 41, 1163-1173.

Öhman, T., Aittola, M., Kostama, V.-P., Raitala, J., Korteniemi, J., Oct. 2008. Polygonal impact craters in Argyre region, Mars: Implications for geology and cratering mechanics. Meteoritics and Planetary Science 43, 1605-1628.

Pearce, S. J., Melosh, H., 1986. Terrace width variations in complex lunar craters. Geophysical Research Letters 13 (13), 1419-1422.

Pike, R. J., 1977. Size-dependence in the shape of fresh impact craters on the Moon. In: Roddy, D. J., Pepin, R. O., Merrill, R. B. (Eds.), Impact and explosion cratering. Pergamon Press, New York, New York, pp. 489-509.

Plesko, C. S., May 2015. Squaring the Circle: Impact Craters as a Diagnostic of Pre-Existing Sub-Surface Target Features. In: Issues in Crater Studies and the Dating of Planetary Surfaces. Vol. 1841 of LPI Contributions. p. 9043.

Poelchau, M. H., Kenkmann, T., Kring, D. A., 2009. Rim uplift and crater shape in Meteor Crater: Effects of target heterogeneities and trajectory obliquity. J. Geophys. Res. 114 (E1).

Pohn, H., Offield, T., 1970. Lunar crater morphology and relative-age determination of lunar geologic units-part 1. classification. US Geological Survey Prof. Paper, 153-162.

Press, W. H., Teukolsky, S. A., Vetterling, W. T., Flannery, B. P., 1988. Numerical Recipes in C, 2nd Edition. Cambridge Univ. Press, Cambridge, UK.

Robbins, S. J., Hynek, B. M., May 2012. A new global database of Mars impact craters $\geq 1 \mathrm{~km}$ : 1. Database creation, properties, and parameters. Journal of Geophysical Research (Planets) 117, 5004

Robinson, M., 2011. LROC WAC global mosaic, LRO-L-LROC-5-RDR-V1.0, WACGLOBAL_256P.

Robinson, M. S., Brylow, S. M., Tschimmel, M., Humm, D., Lawrence, S. J., Thomas, P. C., Denevi, B. W., BowmanCisneros, E., Zerr, J., Ravine, M. A., Caplinger, M. A., Ghaemi, F. T., Schaffner, J. A., Malin, M. C., Mahanti, P., Bartels, A., Anderson, J., Tran, T. N., Eliason, E. M., McEwen, A. S., Turtle, E., Jolliff, B. L., Hiesinger, H., Jan. 2010. Lunar Reconnaissance Orbiter Camera (LROC) Instrument Overview. Space Sci. Rev. 150, 81-124.

Roddy, D. J., 1978. Pre-impact geologic conditions, physical properties, energy calculations, meteorite and initial crater dimensions and orientations of joints, faults and walls at Meteor Crater, Arizona. Lunar Planet. Sci. Conf 9th, 38913930

Ronca, L. B., Salisbury, J. W., 1966. Lunar history as suggested by the circularity index of lunar craters. Icarus 5 , $130-138$.

Schultz, P. H., 1976. Moon morphology: Interpretations based on Lunar Orbiter photography. Vol. 1. Austin, University 
of Texas Press.

Senft, L. E., Stewart, S. T., 2009. Dynamic fault weakening and the formation of large impact craters. Earth and Planetary Science Letters 287 (34), $471-482$

Shoemaker, E. M., 1960. Penetration mechanics of high velocity meteorites, illustrated by Meteor Crater, Arizona. International Geological Congress 21.

Shoemaker, E. M., 1962. Interpretation of lunar craters. In: Kopal, Z. (Ed.), Physics and Astronomy of the Moon. Vol. 538. Academic Press, New York, pp. 283-359.

Tabares-Rodenas, P., Ormö, J., King, D. T., Sep. 2013. Cosmic wabi-sabi: Tell-tale morphological imperfections in impact crater shapes revealed by numerical analysis. Earth and Planetary Science Letters 377, 211-217.

Tanaka, K., Skinner Jr., J., Dohm, J., Irwin III, R., Kolb, E., Fortezzo, C., Platz, T., Michael, G., Hare, T., 2014. Geologic map of Mars. U.S. Geological Survey Scientific Investigations Maps (3292).

Wallis, D., Burchell, M. J., Cook, A. C., Solomon, C. J., McBride, N., 2005. Azimuthal impact directions from oblique impact crater morphology. Monthly Notices of the Royal Astronomical Society 359, 1137-1149.

Wallis, D., McBride, N., 2002. Planetary impact crater analysis with eigenfunction expansion. Mon. Not. Roy. Ast. Soc. $330,458-472$.

Wallis, D., Solomon, C., Kearsley, A., Graham, G., McBride, N., 2002. Modelling radially symmetric impact craters with zernike polynomials. International Journal of Impact Engineering 27 (4), 433 - 457.

Watters, W. A., 2009. Hypervelocity impacts and the evolution of planetary surfaces and interiors. PhD Thesis, Massachusetts Institute of Technology, Cambridge, MA, USA.

Watters, W. A., Geiger, L. M., Fendrock, M., Gibson, R., Feb. 2015. Morphometry of small recent impact craters on Mars: Size and terrain dependence, short-term modification. Journal of Geophysical Research (Planets) 120, 226-254.

Watters, W. A., Grotzinger, J. P., Bell, J., Grant, J., Hayes, A. G., Li, R., Squyres, S. W., Zuber, M. T., Jan. 2011. Origin of the structure and planform of small impact craters in fractured targets: Endurance Crater at Meridiani Planum, Mars. Icarus 211, 472-497.

Weihs, G., Leitner, J., Firneis, M., 2015. Polygonal impact craters on Mercury. Planetary and Space Science 111, 77 82 